\title{
PROSAC: a submillimeter array survey of low-mass protostars
}

\section{The mass evolution of envelopes, disks, and stars from the Class 0 through I stages ${ }^{\star}$}

\author{
J. K. Jørgensen ${ }^{1}$, E. F. van Dishoeck ${ }^{2,3}$, R. Visser ${ }^{2}$, T. L. Bourke ${ }^{4}$, D. J. Wilner ${ }^{4}$, D. Lommen ${ }^{2}$, \\ M. R. Hogerheijde ${ }^{2}$, and P. C. Myers ${ }^{4}$
}

\author{
1 Argelander-Institut für Astronomie, University of Bonn, Auf dem Hügel 71, 53121 Bonn, Germany \\ e-mail: jes@astro.uni-bonn.de \\ 2 Leiden Observatory, Leiden University, PO Box 9513, 2300 RA Leiden, The Netherlands \\ 3 Max-Planck Institut für Extraterrestrische Physik, Giessenbachstrasse 1, 85748 Garching, Germany \\ ${ }^{4}$ Harvard-Smithsonian Center for Astrophysics, 60 Garden Street MS-42, Cambridge, MA 02138, USA
}

Received 14 April 2009 / Accepted 8 September 2009

\section{ABSTRACT}

\begin{abstract}
Context. The key question about early protostellar evolution is how matter is accreted from the large-scale molecular cloud, through the circumstellar disk onto the central star.

Aims. We constrain the masses of the envelopes, disks, and central stars of a sample of low-mass protostars and compare the results to theoretical models for the evolution of young stellar objects through the early protostellar stages.

Methods. A sample of 20 Class 0 and I protostars has been observed in continuum at (sub)millimeter wavelengths at high angular resolution (typically $2^{\prime \prime}$ ) with the submillimeter array. Using detailed dust radiative transfer models of the interferometric data, as well as single-dish continuum observations, we have developed a framework for disentangling the continuum emission from the envelopes and disks, and from that estimated their masses. For the Class I sources in the sample $\mathrm{HCO}^{+} 3-2$ line emission was furthermore observed with the submillimeter array. Four of these sources show signs of Keplerian rotation, making it possible to determine the masses of the central stars. In the other sources the disks are masked by optically thick envelope and outflow emission.

Results. Both Class 0 and I protostars are surrounded by disks with typical masses of about $0.05 M_{\odot}$, although significant scatter is seen in the derived disk masses for objects within both evolutionary stages. No evidence is found for a correlation between the disk mass and evolutionary stage of the young stellar objects. This contrasts the envelope mass, which decreases sharply from $\sim 1 M_{\odot}$ in the Class 0 stage to $\$ 0.1 M_{\odot}$ in the Class I stage. Typically, the disks have masses that are $1-10 \%$ of the corresponding envelope masses in the Class 0 stage and 20-60\% in the Class I stage. For the Class I sources for which Keplerian rotation is seen, the central stars contain 70-98\% of the total mass in the star-disk-envelope system, confirming that these objects are late in their evolution through the embedded protostellar stages, with most of the material from the ambient envelope accreted onto the central star. Theoretical models tend to overestimate the disk masses relative to the stellar masses in the late Class I stage.

Conclusions. The results argue in favor of a picture in which circumstellar disks are formed early during the protostellar evolution (although these disks are not necessarily rotationally supported) and rapidly process material accreted from the larger scale envelope onto the central star.
\end{abstract}

Key words. stars: formation - stars: circumstellar matter - stars: planetary systems: protoplanetary disks - radiative transfer

\section{Introduction}

The fundamental problem in studies of the evolution of lowmass young stellar objects is how material is accreted from the larger scale envelope through the protoplanetary disk onto the central star - and, in particular, what evolutionary time-scales determine the formation and growth of the disk and star and the dissipation of envelopes. Our theoretical understanding of the evolution of YSOs has typically relied on the coupling between theoretical studies and empirical schemes, based, for example, on the spectral energy distributions or colors or on indirect measures, e.g., of the accretion rates. The most commonly used distinction between the embedded Class 0 and I protostars is that

* Appendices are only available in electronic form at http://www . aanda.org the former emit more than $0.5 \%$ of their luminosity at wavelengths longer than $350 \mu \mathrm{m}$. This in turn is thought to reflect that they have accreted less than half of their final mass (e.g., André et al. 1993, 2000), but few hard constraints on the actual evolution of the matter through the embedded protostellar stages exist. Direct measurements of the stellar, disk, and envelope masses during these pivotal stages can place individual protostars in their proper evolutionary context by addressing questions such as what fraction of the initial core mass has been accreted onto the central star, what fraction is being carried away by the action of the protostellar outflows and what fraction has (so-far) ended up in a disk. In this paper, we analyze single-dish and interferometric observations of emission from dust around a sample of 20 Class 0 and I low-mass protostars, as well as observations of a dense gas tracer, $\mathrm{HCO}^{+} 3-2$, for the Class I sources. By 
interpreting continuum data using dust radiative transfer models, we disentangle the contributions from the envelopes and disks to the emission and estimate their masses. Together with stellar masses inferred from Keplerian rotation in resolved line maps (see also Brinch et al. 2007; Lommen et al. 2008), we directly constrain the evolution of matter during the earliest embedded stages of young stellar objects.

The formation of a circumstellar disk is clearly a key event in the evolution of young stellar objects, providing the route through which material is accreted from the large-scale envelope onto the central star. Theoretically a rotationally supported circumstellar disk is expected to form during the collapse of a dense core due to its initial angular momentum: material falling in from the outer regions of the core will be deflected away from the central star into a flat circumstellar disk structure at the "centrifugal radius" where gravity is balanced by rotation (e.g., Ulrich 1976; Cassen \& Moosman 1981; Terebey et al. 1984; Basu 1998). The centrifugal radius will grow in time, with the exact pace depending on the distribution of angular momentum in the infalling core, e.g., whether it can be described as solid-body rotation (e.g., Terebey et al. 1984) or as differential rotation as expected in magnetized infalling cores (e.g., Basu 1998). Even earlier in the evolution of low-mass YSOs, an unstable pseudo-disk may develop as magnetic fields in the core deflect infalling material away from the radial direction toward the central disk plane (Galli \& Shu 1993a,b).

The existence of circumstellar disks has been used to solve the so-called "luminosity problem" (e.g. Kenyon et al. 1990) in which embedded young stellar objects are observed to be underluminous compared to the expected luminosity due to release of gravitational energy under constant mass accretion onto the central star. The solution proposed by Kenyon et al. (1990) is that material is accreted onto the circumstellar disk (and thus at larger radii with a smaller release of gravitational energy) and from there episodically onto the central star, e.g., related to the observed FU Orionis outbursts. The recent compilation of YSO luminosities from the Spitzer Space Telescope "Cores to Disks (c2d)" legacy program (Evans et al. 2009) shows that even the most deeply embedded YSOs are under-luminous compared to the predictions from models - perhaps suggesting that already in these early phases material is not accreted directly on to the central protostar, but rather accumulated either in a circumstellar disk or accreted in another episodic manner.

The main issue about inferring the properties of circumstellar disks around embedded YSOs remains to disentangle the contribution from the larger scale envelopes and circumstellar disks. The continuum emission from dust on the other hand provides an optically thin tracer, whose strength mainly depends on the column density and temperature of the emitting material - and thus potentially provides a direct probe of the dust mass of envelope and/or disks if these can be separated. High angular resolution line observations provide a means to complete the picture of the structure of young stellar objects and their evolution by constraining the dynamical structure of the inner envelope and disk and through that infer the central stellar masses (e.g., Brown \& Chandler 1999; Brinch et al. 2007; Lommen et al. 2008). In particular, aperture synthesis observations at submillimeter wavelengths are important for this goal: the higher excitation transitions of common molecules such as $\mathrm{HCO}^{+}, \mathrm{HCN}$ and CS observed at $1 \mathrm{~mm}$ and shorter wavelengths, probe densities $\geq 10^{5} \mathrm{~cm}^{-3}$ and temperatures $\geq 25 \mathrm{~K}$ (e.g., Evans 1999) and are thus much less sensitive to the structure of the outer envelope/ambient cloud compared to ground-state and low-excitation transitions observable at longer wavelengths.
For more evolved YSOs in fairly isolated regions, singledish continuum observations have been used for comparative studies of statistically significant samples of sources (e.g., Beckwith et al. 1990; André \& Montmerle 1994; Andrews \& Williams 2005, 2007b). Subarcsecond resolution imaging using (sub)millimeter wave interferometers has been done for smaller samples of objects (e.g., Lay et al. 1994; Mundy et al. 1996; Hogerheijde et al. 1997b; Looney et al. 2000). For embedded protostars, the issue arises that both the disks and envelopes may contribute significantly to the emission within the typical single-dish beam and for such sources, interferometric imaging is needed to address the dust distribution on the size scales where disks may be forming. Keene \& Masson (1990) demonstrated the potential of such high angular resolution millimeter observations using data from the OVRO millimeter array: by comparing the interferometric data to models for the collapsing envelope around the L1551-IRS5 Class I protostar, inferred from modeling its SED, Keene \& Masson found that a central unresolved component was required to explain the observed brightness profiles in the interferometric data, such as expected from a central circumstellar disk. In the case of L1551-IRS5, this compact component accounts for about $50 \%$ of the total dust continuum emission - or if interpreted in the context of a circumstellar disk and envelope - a circumstellar disk containing about 20-25\% of the total mass in the system. Hogerheijde et al. (1997a, 1998) used the OVRO Millimeter Array to survey the continuum and line emission at $3 \mathrm{~mm}$ from a sample of 9 Class I protostellar sources. They showed that most of the continuum emission was associated with a compact, unresolved $\left(<3^{\prime \prime}\right)$ component. More recently these kind of studies have seen significant progress with the advent of new data and more detailed dust radiative transfer models (e.g., Hogerheijde \& Sandell 2000; Looney et al. 2003; Jørgensen et al. 2004a, 2005a; Jørgensen 2004; Schöier et al. 2004; Eisner et al. 2005; Brinch et al. 2007; Lommen et al. 2008). In many cases, evidence is found for similarly compact components - although the exact interpretation of their nature still depends on assumptions about the properties of the larger scale envelopes. Still, despite these efforts the studied samples have been limited to at most a handful of sources.

In this paper, we combine continuum and line observations for a large sample of embedded protostars to constrain the masses of their three main components: the envelopes, disks and central stars. Specifically, we discuss single-dish and interferometric observations of a sample of 20 Class 0 and I young stellar objects covering size scales from a few thousand down to a few hundred AU. We use continuum data together with detailed dust radiative transfer to constrain the masses of the larger scale envelopes and circumstellar disks, thereby providing constraints on the evolution of the material through these embedded stages. In addition, we report observations of the $\mathrm{HCO}^{+} 3-2$ line at $267 \mathrm{GHz}$ tracing dense gas in the envelopes around the Class I protostars - in a few cases showing evidence for Keplerian rotation in the inner envelopes/circumstellar disks. This paper is laid out as follows: Sect. 2 introduces the selected samples of Class 0 and I protostars. The details of the new SMA observations of the Class I sources in this sample are presented in Sect. 3; for the Class 0 sources we refer to Jørgensen et al. (2007a). Section 4 discusses the constraints on the envelope and disk properties from the continuum data compared to generic dust radiative transfer models of protostellar envelope. Section 5 discusses the results of the line observations of the Class I sources - in particular, the use of $\mathrm{HCO}^{+} 3-2$ as a tracer of Keplerian motions in the inner envelopes and disks around four of these sources. A discussion of outflow cavities observed in the $\mathrm{HCO}^{+} 3-2$ emission for 
Table 1. Sample of embedded protostars.

\begin{tabular}{llllll}
\hline \hline Source & RA [J2000] & Dec [J2000] & $T_{\text {bol }}[\mathrm{K}]$ & $L_{\text {bol }}\left[L_{\odot}\right]$ & $d$ [pc] \\
\hline L1448-mm & 032538.9 & +304405.4 & 77 & 5.3 & 220 \\
NGC 1333: & & & & & \\
$\quad$-IRAS2A & 032855.6 & +311437.1 & 57 & $20^{a}$ & 220 \\
$\quad$-IRAS4A-SE & 032910.5 & +311331.0 & 43 & 5.8 & 220 \\
-IRAS4A-NW & 032910.4 & +311332.3 & - & - & 220 \\
-IRAS4B & 032912.0 & +311308.1 & 55 & $3.8^{b}$ & 220 \\
-IRAS4B' & 032912.8 & +311307.0 & - & - & 220 \\
L1527 & 043953.9 & +260309.8 & 59 & 2.0 & 140 \\
L483 & 181729.9 & -043939.6 & 60 & 9.0 & 200 \\
B335 & 193700.9 & +073409.7 & 60 & 3.0 & 250 \\
L1157 & 203906.3 & +680216.0 & 42 & 6.0 & 325 \\
& & Class I & & & \\
L1489-IRS & 040442.9 & +261856.3 & 238 & 3.7 & 140 \\
TMR1 & 043913.9 & +255320.6 & 144 & 3.7 & 140 \\
TMC1A & 043935.2 & +254144.4 & 172 & 2.2 & 140 \\
GSS30-IRS3 & 162621.7 & -242250.6 & 300 & $14^{c}$ & 125 \\
GSS30-IRS1 & 162622.2 & -242301.9 & - & - & 125 \\
WL 12 & 162644.2 & -243448.7 & 440 & 2.6 & 125 \\
Elias 29 & 162709.4 & -243720.0 & 350 & 41 & 125 \\
IRS 43 & 162726.9 & -244050.6 & 310 & 6.0 & 125 \\
IRS 54 & 162751.8 & -243145.4 & 740 & 2.5 & 125 \\
IRS 63 & 163135.7 & -240129.6 & 530 & 3.3 & 125 \\
\hline
\end{tabular}

${ }^{a}$ For NGC 1333-IRAS2A Evans et al. (2009) quote a luminosity of $76 L_{\odot}$. Estimating the luminosity from scaling the $70 \mu \mathrm{m}$ flux using the approach of Dunham et al. (2008) gives a luminosity of $19.6 L_{\odot}$, which agrees well with previous estimates from modeling the SED in the range 16-20 $L_{\odot}$. The higher luminosity in the c2d compilation is a result of the SED of IRAS2A being unconstrained at $24 \mu \mathrm{m}$ (Dunham, priv. comm) - and we therefore adopt a luminosity of $20 L_{\odot}$ for this source. ${ }^{b}$ The Spitzer maps of NGC 1333-IRAS4B shows significant emission from a nearby outflow shock within the typical single-dish beam. As total luminosity for this source, we adopt the sum of the two components, SSTc2d J032912.06+311305.4 and SSTc2d J032912.06+311301.7, from Evans et al. (2009). ${ }^{c}$ The emission from GSS30-IRS3 (LFAM1) and GSS30-IRS1 are not separated in the Spitzer catalog (the latter dominates the emission and its luminosity is quoted here).

another three of the Class I sources is deferred to the Appendix where also details of the individual sources are given. Finally, Sect. 6 compares the inferred masses to models for the evolution of the envelope, disk and stellar masses and discusses the assumptions in, e.g., the assumed dust opacities, before Sect. 7 summarizes the main conclusions of the paper.

\section{Sample and data}

\subsection{Sample}

Our data consist of line and continuum observations of 10 Class 0 sources and 9 Class I sources observed as part of the Submillimeter Array (SMA, Ho et al. 2004) ${ }^{1}$ key project, Protostellar Submillimeter Array Campaign (PROSAC, Jørgensen et al. 2007a).

The Class 0 sources were observed in 3 different spectral setups and continuum at $1.3 \mathrm{~mm}(225 \mathrm{GHz})$ and $0.85 \mathrm{~mm}$ ( $345 \mathrm{GHz}$ ) between November 2004 and January 2006, and the data for those were discussed in (Jørgensen et al. 2007a).

The Class I YSOs were selected amongst known Class I sources in the Ophiuchus and Taurus star forming regions also

\footnotetext{
1 The Submillimeter Array is a joint project between the Smithsonian Astrophysical Observatory and the Academia Sinica Institute of Astronomy and Astrophysics, and is funded by the Smithsonian Institution and the Academia Sinica.
}

surveyed by Hogerheijde et al. (1997a) and van Kempen et al. (2009). The sample was constructed to span the expected evolutionary range of Class I sources based on their bolometric temperatures, $T_{\text {bol }}$ (Myers \& Ladd 1993). In total eight fields were selected encompassing 9 YSOs previously classified as embedded Class I protostars. In addition, we include the Class I protostar L1489-IRS, which was observed with the SMA in compact and extended configuration in the same lines as the remaining Class I sources and analyzed using detailed 2D radiative transfer models by Brinch et al. (2007). These surveys provide (sub)millimeter continuum data of $1-3^{\prime \prime}$ resolution with typical sensitivities of a few mJy beam ${ }^{-1}$. The primary beam field of view of the SMA is $43-51^{\prime \prime}$ at $1.1-1.3 \mathrm{~mm}$.

An overview of the total sample is given in Table 1. In this table we give the positions of the sources from fits to the (sub)millimeter continuum position as well as bolometric temperatures and luminosities for the sources - predominantly based on the compilation from the Spitzer/c2d legacy program (Evans et al. 2009).

In addition to the SMA data we collected additional nearinfrared data for each Class I source: Hubble Space Telescope (HST) $1.6 \mu \mathrm{m}$ images from the Near Infrared Camera and MultiObject Spectrometer (NICMOS) camera were obtained from the HST archive at ESO for TMR1, TMC1A, IRS 43 and GSS30. The images were registered using existing near-infrared data (e.g., Barsony et al. 1997). We also include single pointing 


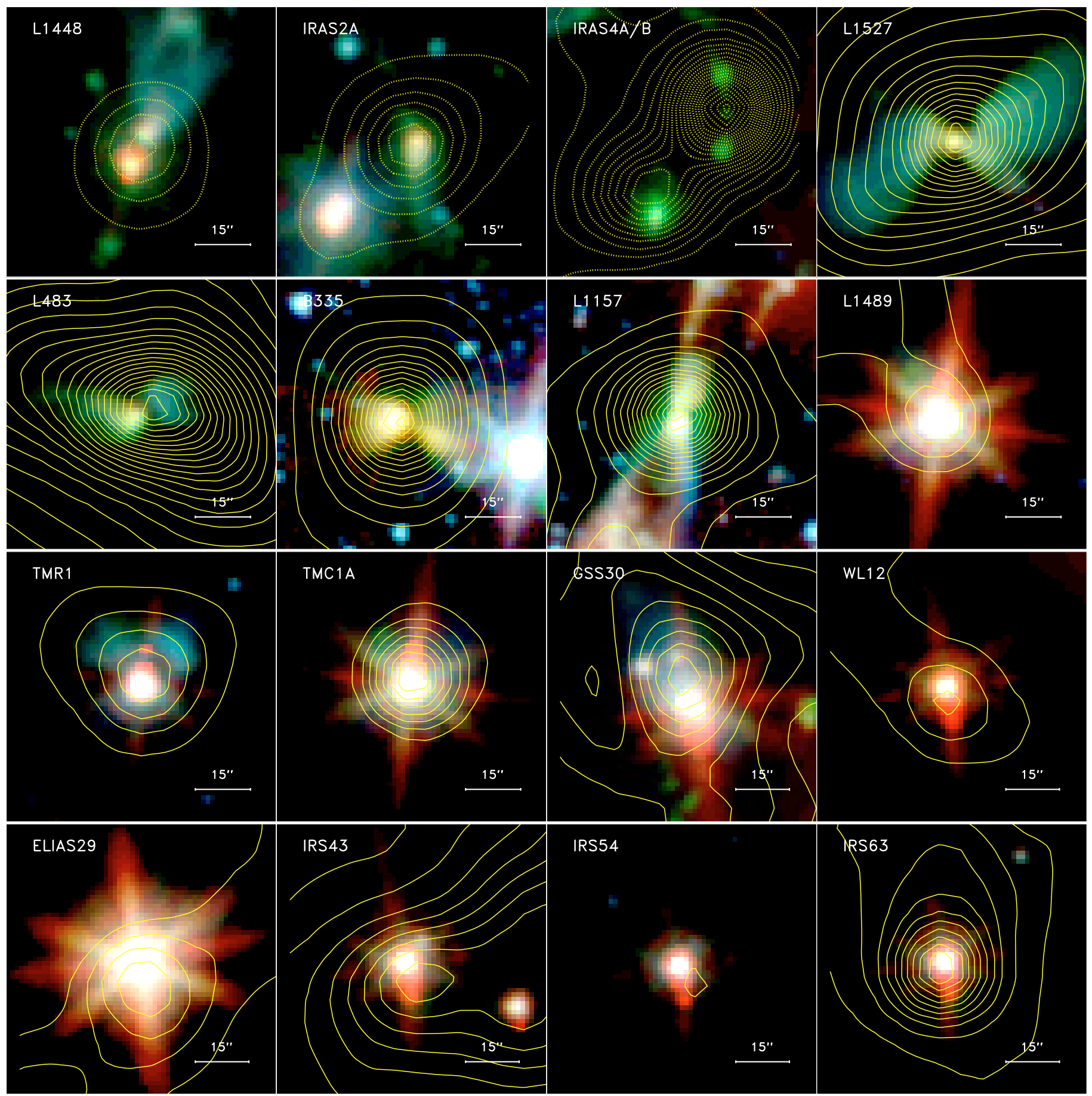

Fig. 1. Spitzer Space Telescope 3.6, 4.5 and $8.0 \mu \mathrm{m}$ images (blue, green and red) of each of our sources with SCUBA $850 \mu \mathrm{m}$ emission (Di Francesco et al. 2008) shown as contours. The contours are given in steps of $0.1 \mathrm{Jy}^{\text {beam }}{ }^{-1}$ - except for L1448, IRAS2A, and IRAS4A/B where the (dotted) contours are given in steps of $0.3 \mathrm{Jy} \mathrm{beam}^{-1}$. For further details about Spitzer observations of individual sources see: Perseus: Jørgensen et al. (2006, 2007b) and Gutermuth et al. (2008), Taurus: Padgett et al. (2008), Ophiuchus: Evans et al. (2009) and Jørgensen et al. (2008), L1527: Tobin et al. (2008), B335: Stutz et al. (2008), and L1157: Looney et al. (2007).

$\mathrm{HCO}^{+} 3-2$ spectra from the James Clerk Maxwell Telescope (JCMT) archive that were originally presented by Hogerheijde et al. (1997a) and unpublished observations that were obtained in connection with the $\mathrm{HCO}^{+} 4-3$ study by van Kempen et al. (2009). For both Class 0 and I sources, recalibrated single-dish submillimeter continuum maps (Fig. 1) were obtained through the JCMT/SCUBA archive legacy project (Di Francesco et al. 2008).

\section{SMA observations of Class I sources}

\subsection{Observations}

The observations of the Class I sources were performed between May 2006 and July 2007 with the Submillimeter Array (SMA) in two variations of its compact configuration. For all observations, all 8 antennas were available in the array, providing projected baselines of 9 to $64 \mathrm{k} \lambda$ (May 2006 and January 2007 

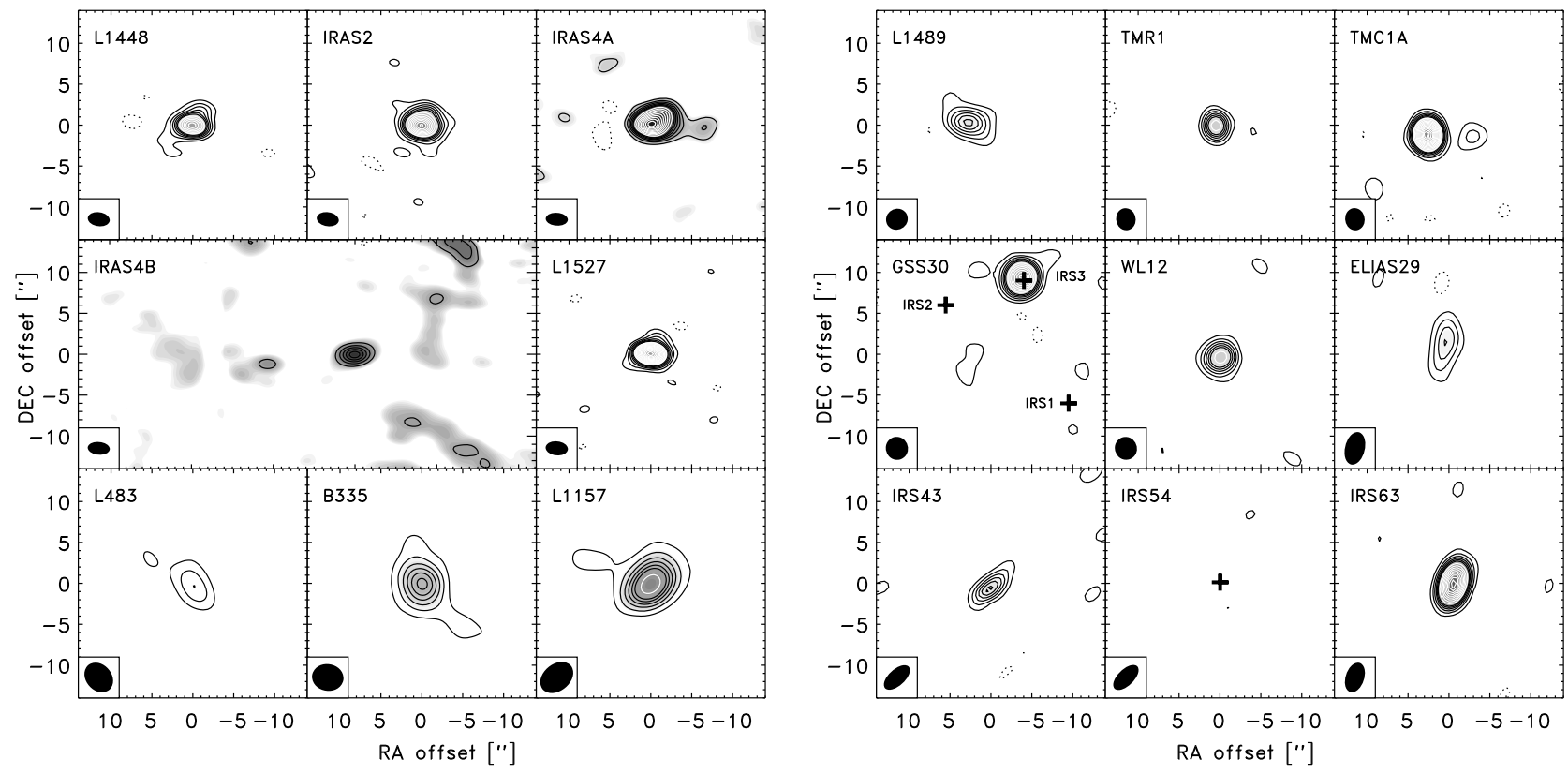

Fig. 2. Overview of the $1.3 \mathrm{~mm}$ continuum emission from the Class 0 sources (left group of panels; see also Jørgensen et al. $2007 \mathrm{a}$ ) and $1.1 \mathrm{~mm}$ continuum emission from the sample of Class I sources (right group of panels; this paper). Contours are given from $3 \sigma$ to $18 \sigma$ in steps of $3 \sigma$ (black contours) and onwards from there in steps of $6 \sigma$ (white contours). In the panels for IRS 54 and GSS30 the Spitzer positions for the known YSOs are indicated with the plus-signs.

observations) and 9 to $104 \mathrm{k} \lambda$ (June and July 2007 observations). Typically, two sources were observed per track with the exception of IRS 63 and Elias 29 that were observed in separate tracks as discussed by Lommen et al. (2008).

The complex gains were calibrated by observations of 1.5-3 Jy quasars typically located within $15^{\circ}$ of the targeted sources once every $20 \mathrm{~min}$. The bandpass was calibrated through observations of strong quasars and planets at the beginning and end of each track. The quasar fluxes were bootstrapped through observations of Uranus with a resulting $\approx 20 \%$ flux calibration uncertainty.

The SMA receivers and correlator was configured to observe the lines of $\mathrm{HCN} J=3 \rightarrow 2(265.886444 \mathrm{GHz})$ and $\mathrm{HCO}^{+} J=3 \rightarrow 2(267.557648 \mathrm{GHz})$ together with the continuum at $1.1 \mathrm{~mm}$ : 1 chunk of 512 channels was centered on each of the lines, resulting in a spectral resolution of $0.2 \mathrm{MHz}$ or $0.23 \mathrm{~km} \mathrm{~s}^{-1}$. The remainder of the $2 \mathrm{GHz}$ bandwidth in each sideband was used to record the continuum.

The initial flagging of the data as well as bandpass, flux and phase/amplitude calibrations were performed in the Mir package (Qi 2008) and subsequent imaging and cleaning in Miriad (Sault et al. 1995). Table 2 summarizes the details of the observations.

\subsection{Continuum data overview}

Figure 2 shows the SMA continuum maps of the embedded protostars in the sample at $1.1 \mathrm{~mm}$ for the Class I sources as well as the $1.3 \mathrm{~mm}$ continuum maps for the Class 0 sources previously presented by Jørgensen et al. (2007a). All sources, except IRS 54, are detected in continuum: one source, TMC1A, shows a fainter secondary component, TMC1A-2 at a separation of 5.5" $(\approx 770 \mathrm{AU})$ whereas the GSS 30 -IRS1, -IRS2, -IRS3 system only shows a continuum detection for GSS30-IRS3.

Most of the observed continuum emission has its origin in the inner few hundred AU of the embedded protostars. This is clearly demonstrated in plots of the observed visibilities as function of projected baseline length (Fig. 3). Most sources are roughly consistent with a point, or marginally resolved, source at the resolution of the SMA. These plots are made using circular averages in the $(u, v)$-plane, i.e., implicitly assume that the emission is spherically symmetric.

To estimate the structure of each source an elliptical Gaussian was fitted to the continuum flux in the $(u, v)$ plane for baselines longer than $20 \mathrm{k} \lambda$, where the contribution from the larger scale collapsing envelope becomes negligible and the plots of visibility amplitude vs. projected baseline length flattens (Fig. 3). The results are given in Table 3. The derived fluxes are slightly lower than the single-dish measurements by Andrews \& Williams $(2005,2007 \mathrm{a})$ extrapolated to $1.1 \mathrm{~mm}$ assuming optical thin dust with $\kappa \propto \nu^{\beta}$ and $\beta=1-$ but still more than $50 \%$ of the total flux for each source is recovered. This suggests that although the envelope itself contributes on scales smaller than the approximately $15^{\prime \prime}$ of the JCMT beam, the flux from the central compact component dominates. The sources that show the biggest discrepancies between the single-dish fluxes and interferometric fluxes (IRS 63, WL 12, GSS30 and Elias 29) are those that show clear evidence of extended emission in Fig. 1. The deconvolved sizes of the continuum sources from the Gaussian fits suggest that they represent structures with sizes of up to 250-300 AU diameter.

\subsection{Line data overview}

Figure 4 shows an overview of the $\mathrm{HCO}^{+} 3-2$ spectra toward the continuum positions of each source from the interferometric observations, as well as single-dish spectra from the JCMT archive. The $\mathrm{HCO}^{+} 3-2$ line profiles vary significantly in strength between different sources, but also relatively between the single-dish and interferometric data. In the singledish data all sources show $\mathrm{HCO}^{+} 3-2$ lines with intensities of $1.5-10 \mathrm{~K} \mathrm{~km} \mathrm{~s}^{-1}$, but in the interferometer data only 6 of the 9 Class I sources have clearly detected lines. IRS 43 shows 
Table 2. Log of observations of Class I sources.

\begin{tabular}{lllll}
\hline \hline Sources & Date & Gain calibrator (flux) & Beam size & Continuum rms \\
\hline IRS 63 & 2006 May 15 & J1626-298 (1.3 Jy), J1517-243 (2.4 Jy) & $4.0^{\prime \prime} \times 2.3^{\prime \prime}$ & $5.5 \mathrm{mJy} \mathrm{beam}^{-1}$ \\
Elias 29 & 2006 May 17 & $\mathrm{J} 1626-298(1.7 \mathrm{Jy}), \mathrm{J} 1517-243(3.2 \mathrm{Jy})$ & $4.0^{\prime \prime} \times 2.3^{\prime \prime}$ & $3.6 \mathrm{mJy} \mathrm{beam}^{-1}$ \\
TMR1, TMC1A & 2007 Jan 02 & $\mathrm{J} 0530+135(2.8 \mathrm{Jy}), 3 \mathrm{c} 111(2.3 \mathrm{Jy})$ & $2.7^{\prime \prime} \times 2.4^{\prime \prime}$ & $2.6 \mathrm{mJy} \mathrm{beam}^{-1}$ \\
$\mathrm{IRS} \mathrm{43,} \mathrm{IRS} \mathrm{54}$ & 2007 Jun 27 & $\mathrm{J} 1626-298(1.5 \mathrm{Jy}), \mathrm{J} 1517-243(1.6 \mathrm{Jy})$ & $3.9^{\prime \prime} \times 2.0^{\prime \prime}$ & $2.8 \mathrm{mJy} \mathrm{beam}^{-1}$ \\
$\mathrm{WL} \mathrm{12,} \mathrm{GSS30}$ & 2007 Jul 04 & $\mathrm{J} 1626-298(1.3 \mathrm{Jy}), \mathrm{J} 1517-243(1.4 \mathrm{Jy})$ & $2.8^{\prime \prime} \times 2.7^{\prime \prime}$ & $2.6 \mathrm{mJy} \mathrm{beam}^{-1}$ \\
\hline
\end{tabular}

Table 3. Results of elliptical Gaussian and point source fits to the continuum visibilities for the Class I sources.

\begin{tabular}{llllll}
\hline \hline Source & \multicolumn{2}{c}{ Position } & \multicolumn{2}{c}{ Size $^{a}, F W H M$} & \multicolumn{2}{c}{ Flux (density) } \\
& $\begin{array}{l}\text { RA } \\
(\mathrm{J} 2000.0)\end{array}$ & $\begin{array}{l}\text { Dec } \\
(\mathrm{J} 2000.0)\end{array}$ & $\begin{array}{l}\theta_{1.1 \mathrm{~mm}} \\
{\left[{ }^{\prime \prime}\right]}\end{array}$ & $\begin{array}{l}F_{1.1 \mathrm{~mm}}{ }^{b} \\
{[\mathrm{mJy}]}\end{array}$ & $\begin{array}{l}S_{850 \mu \mathrm{m}}\left(15^{\prime \prime}\right)^{c} \\
{\left[\mathrm{mJy} \mathrm{beam}^{-1}\right]}\end{array}$ \\
\hline TMR1 & 043913.91 & +255320.63 & $\ldots{ }^{d}$ & $79^{d}$ & 480 \\
TMC1A & 043935.20 & +254144.35 & $0.69 \times 0.40$ & $256(246)$ & 780 \\
TMC1A-2 & 043934.80 & +254143.87 & $3.7 \times 1.1$ & $48(26)$ & $\ldots$ \\
IRS 43 & 162726.91 & -244050.62 & $1.9 \times 0.13$ & $75(56)$ & 990 \\
IRS 54 & $162751.80^{d}$ & $-243145.4^{d}$ & $\ldots$ & $\ldots$ & $\ldots$ \\
IRS 63 & 163135.65 & -240129.55 & $0.63 \times 0.0096$ & $473(459)$ & 1220 \\
GSS30-IRS3 & 162621.71 & -242250.63 & $1.4 \times 1.3$ & $204(144)$ & 990 \\
WL 12 & 162644.19 & -243448.74 & $1.9 \times 1.5$ & $104(64)$ & 360 \\
Elias 29 & 162709.44 & -243719.99 & $5.8 \times 1.8$ & $109(48)$ & 590 \\
\hline
\end{tabular}

${ }^{a}$ Deconvolved size from Gaussian fit. ${ }^{b}$ Flux density from Gaussian fit to SMA data in the $(u, v)$-plane. Number in parentheses from point source fit. ${ }^{c}$ Peak flux at $850 \mu \mathrm{m}$ in JCMT $15^{\prime \prime}$ single-dish beam. ${ }^{d}$ TMR1 unresolved in SMA observations. Only result from point source fit listed. ${ }^{e}$ IRS 54 undetected at the SMA; position from Spitzer Space Telescope observations.

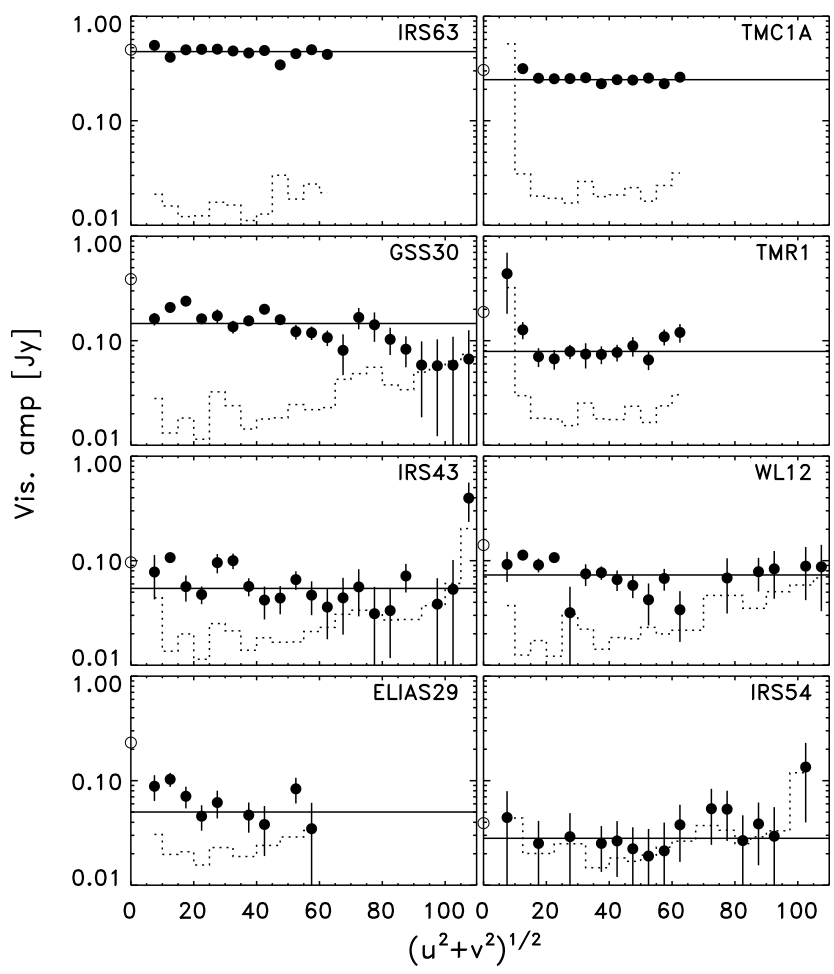

Fig. 3. Plot of the continuum flux as function of projected baseline length. The dotted histograms indicate the zero-amplitude signal, i.e., the anticipated signal in the absence of source emission. The open circles indicate the single-dish peak flux $\left[\right.$ in $\mathrm{Jy} \mathrm{beam}^{-1}$ ] from the JCMT/SCUBA maps toward each source extrapolated to $1.1 \mathrm{~mm}$.

the highest intensity and broadest line profile in the $\mathrm{HCO}^{+}$ SMA observations. Interestingly, the interferometric spectra toward TMR1 and TMC1A only probe the blue-shifted emission relative to the single-dish systemic velocity. Figure 5 shows SMA maps of the $\mathrm{HCO}^{+}$and $\mathrm{HCN}$ emission integrated over 4 velocity intervals around the systemic velocity. Again, clear differences are found between the sources. IRS 63 and IRS 43 show red- and blue-shifted emission around the continuum position whereas TMR1, TMC1A and GSS30-IRS1 display emission extended from one side relative to the source position. Toward Elias 29 a complex structure with large-scale extended $\mathrm{HCO}^{+}$emission from the nearby ridge is seen. For that source material with the most extreme red/blue-shifted velocities around the continuum position trace the Keplerian rotation in the inner envelope/disk (see Lommen et al. 2008).

\section{Analysis of continuum data: disk vs. envelope masses}

With large continuum surveys at hand the first step is to disentangle the envelope and disk contributions to the dust continuum emission and from this derive the disk and envelope masses. Ideally fully self-consistent models can be derived for all sources taking into account the full spectral energy distributions as well the interferometric data (e.g., Jørgensen et al. 2005a; Lommen et al. 2008). However, much information can be derived from just simple comparisons of the single-dish and interferometric fluxes.

As discussed by, e.g., Terebey et al. (1993) the continuum emission from a cloud core or circumstellar disk is well approximated by low optical depth in which case the intensity profile is simply found by integrating the temperature and density profile of the core or disk along the line of sight (e.g., Eq. (1) of Terebey et al. 1993). The complication naturally arises that both the density and temperature vary throughout protostellar disks and envelopes. Dust radiative transfer models calculate the dust temperature distribution self-consistently and furthermore produce images at different wavelengths, which can be directly compared to the observations. 

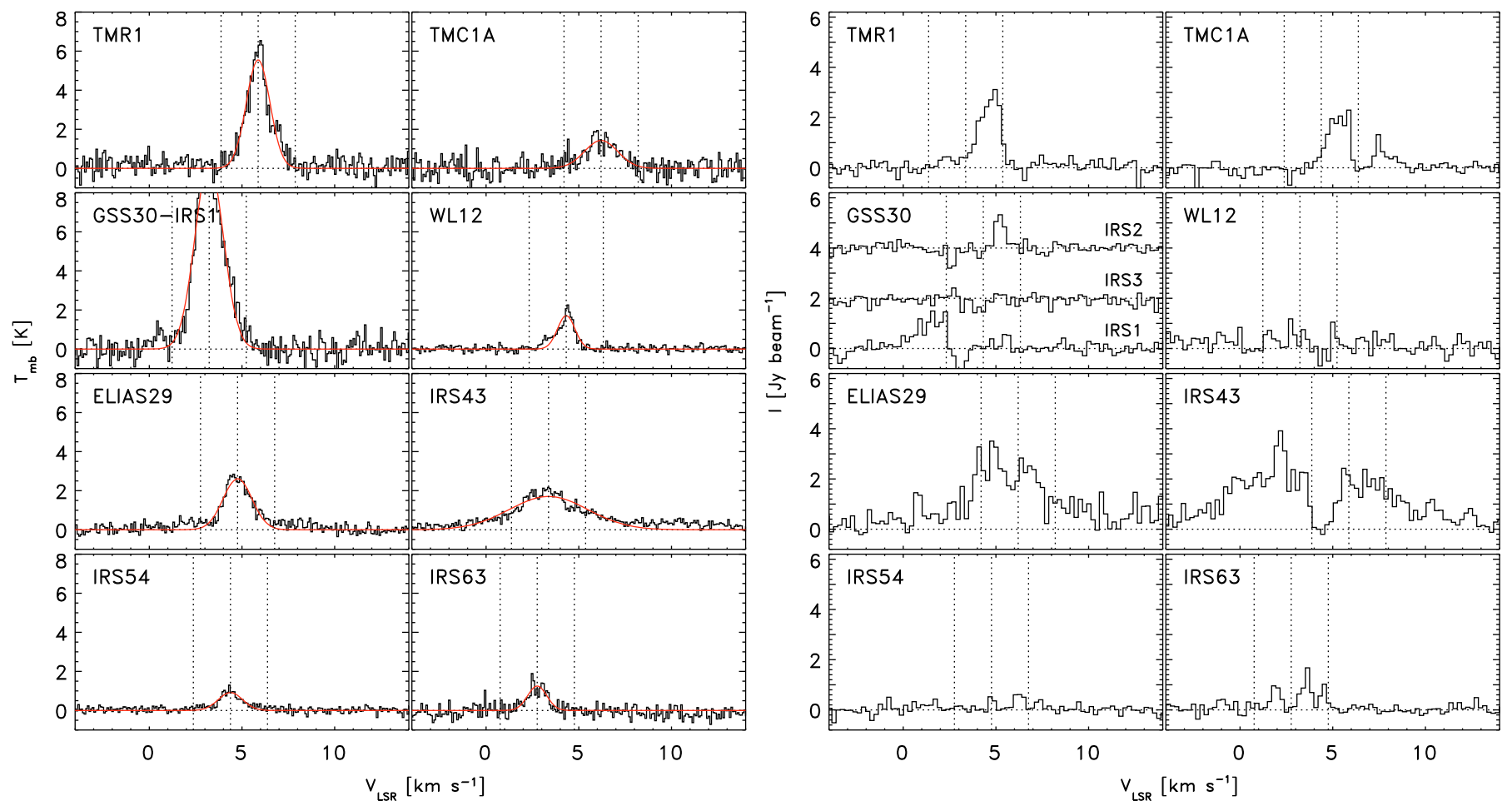

Fig. 4. $\mathrm{HCO}^{+} 3-2$ single-dish spectra from the JCMT archive (left) and toward the continuum positions of each source from the SMA observations $\left(\right.$ right). A Gaussian was fitted to each single-dish spectrum for each source and the centroid velocity as well as velocities $\pm 2 \mathrm{~km} \mathrm{~s}^{-1}$ from this are shown with dotted lines in each panel and used to calculate moment maps in Fig. 5.
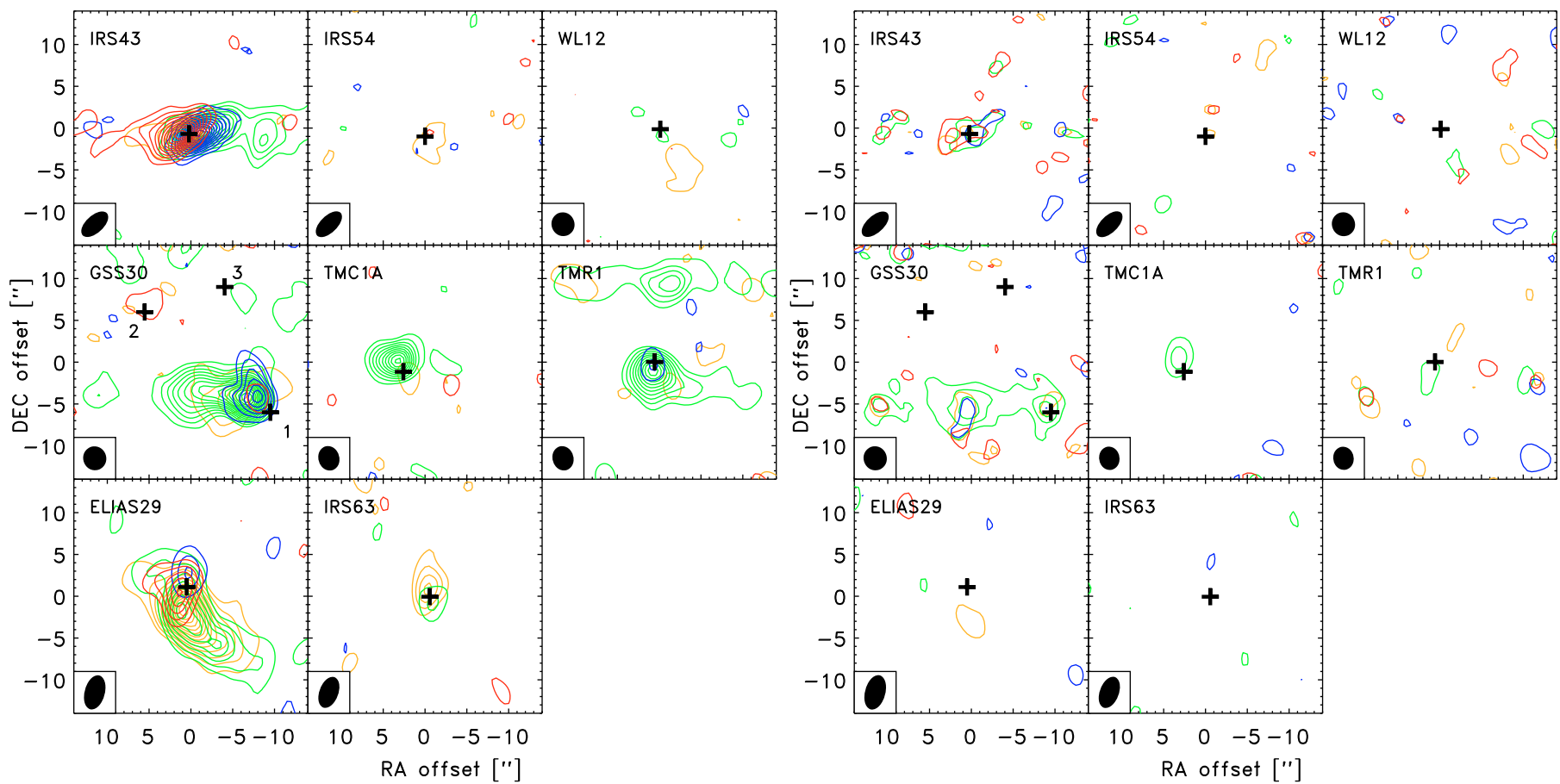

Fig. 5. Overview of the $\mathrm{HCO}^{+} 3-2$ (left) and $\mathrm{HCN} 3-2$ (right) emission from the sample of Class I sources. The contours (shown in $3 \sigma$ intervals) indicate emission integrated over intervals from -4 to $-2 \mathrm{~km} \mathrm{~s}^{-1}$ (blue), -2 to $0 \mathrm{~km} \mathrm{~s}^{-1}$ (green), 0 to $+2 \mathrm{~km} \mathrm{~s}^{-1}$ (orange) and +2 to $+4 \mathrm{~km} \mathrm{~s} \mathrm{k}^{-1}$ (red) relative to the systemic velocities from the Gaussian fits to the single-dish spectra.

In this section we use such dust radiative transfer models to derive the masses of the envelopes and disks around the sample of Class 0 and I protostars. We first explore one-dimensional dust radiative transfer models of protostellar envelopes: we compare the emission from a spherically symmetric envelope around a central source of heating (i.e., protostar) at the scales observed with single-dish telescopes compared to our interferometric observations (Sect. 4.1) and furthermore use the models to establish a direct relationship between the single-dish submillimeter flux and mass of an envelope around a given protostar as 
function of its luminosity and distance (Sect. 4.2). Finally, we use these results to separate the envelope and disk emission from the envelopes and disks and estimate their masses for each of the objects in our sample (Sects. 4.3 and 4.4).

\subsection{Dust radiative transfer models; how much does an envelope contribute to the compact emission?}

First, to test the hypothesis that most of the flux in the interferometric data at baselines of $50 \mathrm{k} \lambda$ is in fact from the central compact component we calculated a set of self-consistent models for the dust in protostellar envelopes using the Dusty radiative transfer code (Ivezić et al. 1999). Dusty solves the temperature profile for a spherically symmetric envelope around a central source of heating given the power-law envelope density profile, input spectrum of the central heating source and dust properties. The code furthermore calculates images at user-specified wavelengths, which can be compared to observed images to iteratively place constraints on, e.g., the density profile. It has previously been used to constrain the physical structures of the envelopes around a large sample of deeply embedded protostars (Jørgensen et al. 2002).

We fixed the effective temperature of the central black-body to $1500 \mathrm{~K}$ mimicking the combined contribution from a low effective temperature young star and a disk. Since most of this emission is reprocessed in the larger-scale cold envelope, the exact shape and temperature of the input spectrum does not affect the distribution and strength of the emerging submillimeter emission (see also Jørgensen et al. 2002, 2005b; Schöier et al. 2002; Shirley et al. 2002). We furthermore fixed the outer envelope radius to $8000 \mathrm{AU}$ (typical of envelopes in clustered regions such as Ophiuchus and Perseus) and adopted envelope density profiles with $n \propto r^{-p}$ with $p=1.5$. Such density profiles are expected for free-falling envelopes, e.g., within the collapse expansion wave in the inside-out collapse model of Shu (1977). Detailed models of envelopes of Class 0 and I sources constrained by SCUBA mapping observations find density profiles with $p=1.5-2.0$ (e.g., Jørgensen et al. 2002; Shirley et al. 2002; Young et al. 2003). Those models do not include the contributions from a central disk, which can steepen the derived density profile index artificially by $0.2-0.5$. A power-law index of $1.5-1.8$ for the envelope density profile therefore appears to be consistent with the envelope structure at these scales. As in previous work, we adopt the dust opacity law of Ossenkopf \& Henning (1994) for grains with thin ice mantles, coagulated at a density of $n_{\mathrm{H}_{2}} \sim 10^{6} \mathrm{~cm}^{-3}$. This dust opacity law has $\kappa_{850 \mu \mathrm{m}}=0.0182 \mathrm{~cm}^{2} \mathrm{~g}^{-1}$ (dust+gas) and scales approximately with frequency as $\kappa_{v} \propto v^{1.7}$ at $1 \mathrm{~mm}$. Since we for now just deal with the fluxes at submillimeter wavelengths, the derived masses can be directly rescaled using a different dust opacity law.

With these fixed parameters we then calculated a set of dust radiative transfer models varying the inner radius, luminosity and mass of the envelope. We tested models with inner radii, $R_{i}=10,25,50,100,200 \mathrm{AU}$, envelope masses between 0.01 and $0.5 M_{\odot}$ and luminosities of 1 and $5 L_{\odot}$. Together these parameter ranges cover models representative for the majority of our sources.

For each model, the radiative transfer code calculates the temperature profile self-consistently as well as images at the wavelengths of our single-dish and interferometric observations $(850 \mu \mathrm{m}$ and $1.1 \mathrm{~mm}$, respectively). The images at the wavelength of our interferometric data were subsequently multiplied by the SMA primary beam and Fourier transformed,
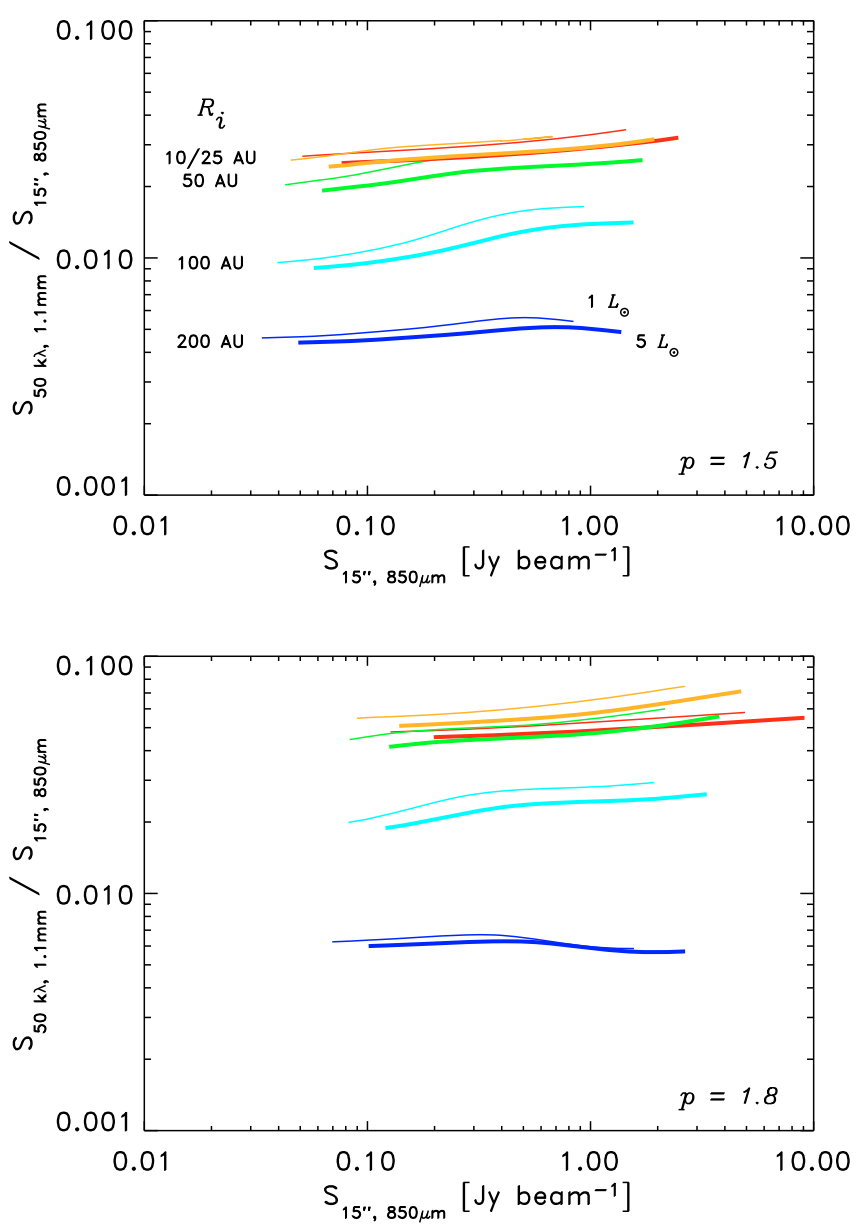

Fig. 6. Ratio of interferometric fluxes ( $1.1 \mathrm{~mm}$ flux at $50 \mathrm{k} \lambda ; S_{50 k \lambda, 1.1 \mathrm{~mm}}$ ) and single-dish fluxes $\left(850 \mu \mathrm{m}\right.$ peak flux in $15^{\prime \prime}$ SCUBA beam; $\left.S_{15^{\prime \prime}, 850 \mu \mathrm{m}}\right)$ as function of single-dish flux for a set of models varying the inner radius (10 AU, $25 \mathrm{AU}, 50 \mathrm{AU}, 100 \mathrm{AU}$ and $200 \mathrm{AU}$ shown with red, orange, green, light blue and dark blue lines, respectively; see also upper panel), source luminosity ( 1 and $5 L_{\odot}$ shown with thin and thick lines, respectively) and envelope masses (along each model line). Envelopes have density profiles, $n \propto r^{-p}$ with $p=1.5$ (upper) and $p=1.8$ (lower) and masses ranging from $0.01 M_{\odot}$ at low $S_{15^{\prime \prime}}$ to $0.5 M_{\odot}$ at high $S_{15^{\prime \prime}}$.

mimicking the interferometric observations. From these observations we extracted the flux at baselines of $50 \mathrm{k} \lambda\left(3.5^{\prime \prime}\right.$ diameter) as an estimate of the contribution to the SMA $1.1 \mathrm{~mm}$ flux from the envelope. Likewise, images at $850 \mu \mathrm{m}$ were convolved with the SCUBA beam and the peak flux in the central beam was extracted as an estimate of its single-dish $850 \mu \mathrm{m}$ peak flux.

Figure 6 shows the ratio of the $1.1 \mathrm{~mm}$ interferometric fluxes at baselines of $50 \mathrm{k} \lambda$ and single-dish peak fluxes at $850 \mu \mathrm{m}$ as function of single-dish peak flux. As shown, the contribution of the envelope to $1.1 \mathrm{~mm}$ emission at baselines of $50 \mathrm{k} \lambda$ is at most only $4 \%$ of the modeled $850 \mu \mathrm{m}$ peak flux. If the envelope is steeper, $n \propto r^{-1.8}$, which seems to be the steepest envelope structure supported by the modeling of the single-dish data, the envelope contribution to the $1.1 \mathrm{~mm}$ emission at baselines of $50 \mathrm{k} \lambda$ increases to $8 \%$ of the peak single-dish flux at $850 \mu \mathrm{m}$.

As expected models with large inner radii resolved by the interferometer show less compact flux lowering the ratio of the interferometer-to-single-dish flux. This increase in flux with decreasing inner radius reaches its maximum of $4 \%$ for an inner 


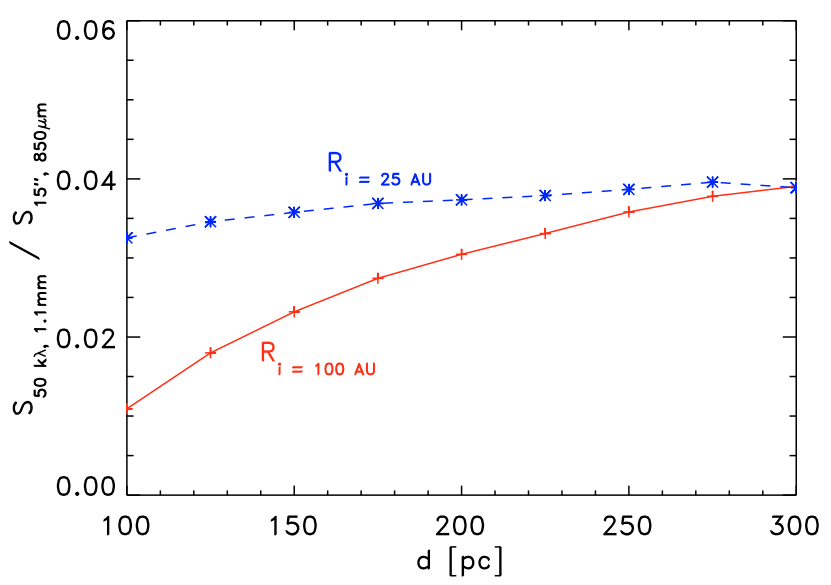

Fig. 7. Ratio between the interferometer flux at $1.1 \mathrm{~mm}$ and single-dish beam peak flux at $850 \mu \mathrm{m}$ as function of distance to the source for an envelope with $p=1.5, R_{\text {out }}=8000 \mathrm{AU}, R_{\mathrm{i}}=25 \mathrm{AU}$ (dashed line) and $100 \mathrm{AU}$ (solid line) and an envelope mass $\approx 0.3 M_{\odot}$.

radius of about $25 \mathrm{AU}$. Material at smaller inner radii is not probed by the existing interferometric observations: this reflects the distribution of mass in the power-law envelope ensuring that most mass is at large scales, or that most of the dust continuum emission is due to mass located at the typical size scale probed by the interferometer.

Using these results we can estimate an upper limit to the envelope contribution at $50 \mathrm{k} \lambda$ for the observed sources by taking $4 \%$ of the $850 \mu \mathrm{m}$ JCMT peak flux and comparing that directly to the observed $1.1 \mathrm{~mm}$ interferometric flux at $50 \mathrm{k} \lambda$ : the ratio between these two numbers then gives the relative envelope contribution to the $1.1 \mathrm{~mm}$ interferometric data. This comparison confirms that the $1.1 \mathrm{~mm}$ SMA measurements of the Class I sources (Table 3) predominantly contain emission from the central disk: in median, $87 \%$ of the observed interferometer flux is from the disk, as also suggested by their flat visibility amplitudes as function of projected baseline length in Fig. 3. For comparison, the Class 0 sources have a median disk contribution of $68 \%$ of their observed interferometer flux. The results in Fig. 6 are shown for a distance of $125 \mathrm{pc}$. The ratio between the interferometer and single-dish peak flux measures the relative surface brightnesses on the spatial scales probed by each and does not change significantly when the distance increases - as long as the inner envelope cavity is not large enough to be resolved (Fig. 7).

\subsection{Calibration of envelope mass - continuum flux relation}

With a separation of the envelope and disk contribution to the single-dish and interferometric observations, the next task is to derive masses from each component using the observed fluxes. For prestellar cores it is usually a good approximation to assume that their dust is optically thin and isothermal with a temperature of $10-15 \mathrm{~K}$ and from that estimate the mass of the core from single-dish observations of their submillimeter continuum fluxes. However, an envelope with an internal heating source, such as a central protostar, will have a distribution of temperatures - which will strongly depend on the source luminosity.

A way to circumvent these problems is again to use the results from the detailed dust radiative transfer models: Fig. 8 compares the relations between the envelope mass, peak flux and
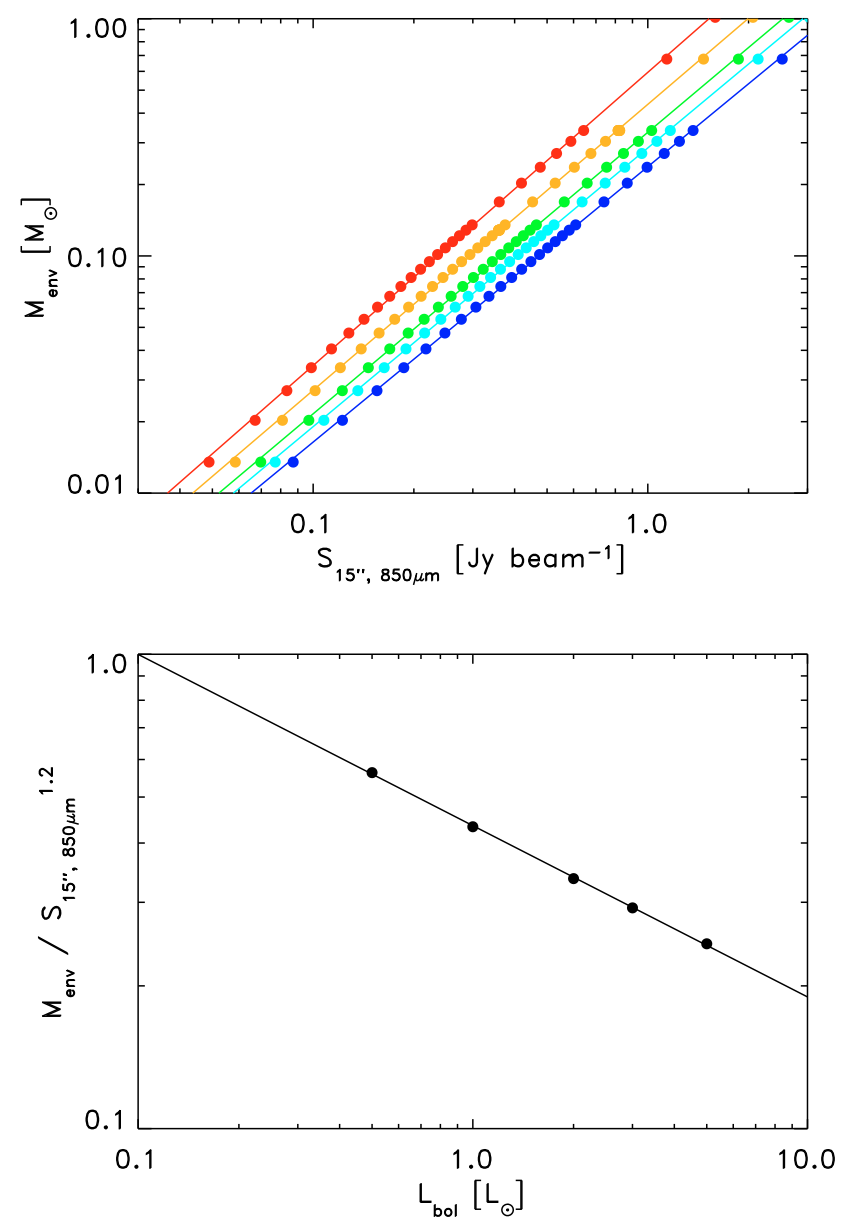

Fig. 8. Results of dust radiative transfer calculations for simple envelope models at $d=125 \mathrm{pc}$. Upper: the relation between envelope mass and peak flux in the JCMT beam for envelope models with $0.5,1,2,3$ and $5 L_{\odot}$ from left (red) to right (blue): each point corresponds to a model in our grid with increasing line-of-sight optical depths at $100 \mu \mathrm{m}$ ranging from 0.01 to 0.20 in steps of 0.01 , from 0.20 to 0.50 in steps of 0.05 and at 1.0. Overplotted also are power-law fits with $M_{\text {env }} \propto S_{15^{\prime \prime}, 850 \mu \mathrm{m}}^{1.2}$. Lower: relation between the $M_{\text {env }} / S_{15^{\prime \prime}, 850 \mu \mathrm{m}}^{1.2}$ normalization factor and luminosity. Overplotted is the best fit $M_{\text {env }} / S_{15^{\prime \prime}, 850 \mu \mathrm{m}}^{1.2} \propto L_{\text {bol }}^{0.36}$ powerlaw relation between these quantities.

luminosity for the protostellar envelopes determined from the radiative transfer modeling. As shown these dust radiative transfer models give rise to simple power-law relationships between the envelope mass and observables which can be expressed as:

$M_{\mathrm{env}}=0.44 M_{\odot}\left(\frac{L_{\mathrm{bol}}}{1 L_{\odot}}\right)^{-0.36}\left(\frac{S_{15^{\prime \prime}, 850 \mu \mathrm{m}}}{1 \mathrm{Jy} \mathrm{beam}^{-1}}\right)^{1.2}\left(\frac{d}{125 \mathrm{pc}}\right)^{1.2}$.

Together with the luminosity, the above expression makes it possible to evaluate the mass of each protostellar envelope from its peak flux only - once the contribution from the disk emission to the single-dish flux has been subtracted (see also Sect. 4.3). This relation is particularly useful in crowded regions where it is possible to estimate the peak flux of a given source, but where it is difficult to evaluate its outer radius and thus its extended flux due to confusion. The luminosity can either be derived from the full spectral energy distribution of each source or by using the empirical correlation between source internal luminosity and $70 \mu \mathrm{m}$ Spitzer flux by Dunham et al. (2008). 
These results are generally in good agreement with the models discussed by Terebey et al. (1993): they find a relationship between the flux and luminosity and distance which for a given envelope mass scales roughly as $F \propto L^{0.2} d^{-0.7}$ compared to our results which for a given envelope mass are equivalent to $F \propto L^{0.3} d^{-1.0}$. The different slope with respect to distance is caused by the slightly flatter density profile predicted by the Terebey et al. (1984) model: for a power-law envelope similar to ours, Terebey et al. (1993) find that $F \propto d^{-0.9}$. The remaining difference in the exponent of this relation as well as the difference in the exponents for the luminosity is caused by the envelopes being slightly optically thick to their own radiation which increases the temperature (and thus emission) on small scales and causes the underlying temperature profile to depart from the single power-law applicable for an envelope fully optically thin to its own radiation.

The derived expressions are particularly useful for interpreting large samples of sources detected, e.g., through the largescale submillimeter maps provided by ongoing and upcoming surveys with bolometer arrays such as LABOCA and SCUBA 2. One of the main goals of those surveys are naturally to determine core masses and this is often done using single isothermal cores with two temperatures: one for cores with and one without embedded protostellar sources (e.g., Enoch et al. 2007; Hatchell \& Fuller 2008). However, with Eq. (1) we can take the dependence of the source submillimeter flux on the luminosity into account: according to Eq. (1) a factor 2.3 decrease in mass is expected due to the increase of the luminosity by a factor 10 for the same peak flux - or put in another way: if the mass for a $1 L_{\odot}$ core is derived using a temperature of $15 \mathrm{~K}$, that for a $10 L_{\odot}$ protostellar core should adopt a temperature of $23.5 \mathrm{~K}$. Assuming a similar temperature for all protostellar cores would therefore lead to a mass-luminosity relation, $L \sim M^{2.8}$. This is slightly steeper but close to the relation found by Hatchell et al. (2007) with $L \sim M^{1.96}$ with an uncertainty of \pm 0.36 in the power-law index of the observationally derived relation.

\subsection{Determining envelope and disk masses from continuum measurements}

With the resuts from the previous section we can now compare the derived compact and single-dish fluxes across the sample. The Class 0 and I sources were not observed at exactly the same wavelengths at the SMA. The Class 0 sources were observed at $1.3 \mathrm{~mm}(230 \mathrm{GHz})$ and $0.8 \mathrm{~mm}(345 \mathrm{GHz})$ whereas the Class I sources discussed here were observed at $1.1 \mathrm{~mm}$ $(270 \mathrm{GHz})$. Class 0 and I sources have been observed at $850 \mu \mathrm{m}$ with SCUBA on the JCMT. To take this difference into account we interpolate the Class 0 point source fluxes estimated on baselines longer than $40 \mathrm{k} \lambda$ from Jørgensen et al. (2007a) using the derived spectral slope of 2.5 from that paper, i.e., less steep than for the envelope. With this correction the fluxes of each source at similar angular scales are compared directly (Fig. 9).

To estimate the masses of both components, a few more steps are required: both the envelope and disk contribute to the observed emission in both the single-dish and interferometer beams. However, with the above results we can quantify the maximum contribution from the envelope on the $1.1 \mathrm{~mm}$ interferometric flux at $50 \mathrm{k} \lambda$ as no more than about $4 \%$ of the envelope single-dish peak flux at $850 \mu \mathrm{m}$. Using this upper limit, we can write the two equations with the envelope flux at $850 \mu \mathrm{m}$, $S_{\text {env }}$, and disk flux at $1.1 \mathrm{~mm}, S_{\text {disk }}$ as two unknowns that can easily be solved for:

$S_{50 \mathrm{k} \lambda}=S_{\mathrm{disk}}+c \cdot S_{\mathrm{env}}$

$S_{15^{\prime \prime}}=S_{\text {disk }}(1.1 / 0.85)^{\alpha}+S_{\text {env }}$.

In these equations $\alpha$ is the spectral index ${ }^{2}$ of the dust continuum emission at millimeter wavelengths in the disks found to be $\approx 2.5$ from the observations of the Class 0 sources in our sample (Jørgensen et al. 2007a). $c$ is the fraction of the envelope $850 \mu \mathrm{m}$ single-dish peak flux observed at $1.1 \mathrm{~mm}$ by the interferometer at $50 \mathrm{k} \lambda$. For this discussion we adopt the upper limit to $c=0.04$ from the above discussion for $p=1.5$. We note that this contribution generally is small enough that the direct measurement by the interferometer is a good first estimate of the actual compact flux not associated with the infalling $\left(n \propto r^{-1.5}\right)$ envelope.

To derive envelope masses, a few additional effects need to be taken into account here: as shown above the envelope masses scale with distance as $d^{1.2}$ for a fixed peak flux, whereas the disk masses (largely unresolved) are expected to scale as $d^{2}$. In addition, since the submillimeter continuum emission measures the dust mass weighted by temperature, the derived envelope masses are found to depend on the luminosity (see, Eq. (1)). Taking this into account, however, it is possible to directly estimate the masses of the envelopes from the observed fluxes once the contribution from the disk has been estimated through Eqs. (2) and (3) (Table 4). For the disk masses we assume that the dust continuum emission is optically thin and coming from an isothermal disk with a temperature of $30 \mathrm{~K}$ and a dust opacity at $1.1 \mathrm{~mm}$ similar to that of the dust in the protostellar envelopes. We return a discussion of these assumptions in Sect. 6.1

\subsection{Resulting disk and envelope masses}

Figure 9 compares the interferometric and single-dish fluxes scaled to $1.1 \mathrm{~mm}$ as well as disk and envelope masses for all the sources in our sample. As expected the deeply embedded, Class 0, protostars have larger fluxes in the single-dish relative to the smaller interferometric beam, reflected in a lower $S_{50 \mathrm{k} \lambda} / S_{15^{\prime \prime}}$ ratio (upper left panel of Fig. 9). A similar effect was noted by Ward-Thompson (2007) who compared interferometric and single-dish fluxes at $850 \mu \mathrm{m}$ from literature studies of a sample of 9 YSOs (6 Class 0, 2 Class I and 1 Class II sources). Taken by itself this can naturally reflect one or two effects: (i) the envelope masses for these sources are larger also resulting in their redder SEDs or; (ii) the disk masses are smaller for the more deeply embedded protostars (e.g., if significant disk growth occurs from the Class 0 through I stages). The interferometric fluxes do not show such an increase going from the Class 0 to I stages, however (upper right panel of Fig. 9). This argues against a significant increase in disk masses being the explanation.

The lower panels of Fig. 9 compare the envelope and disk masses for all the sources in the Class 0 and I samples as a function of bolometric temperature. Again, the disk/envelope mass ratio is seen to be lower for the more deeply embedded Class 0 protostars reflecting their higher envelope masses at comparable disk masses as those in the Class I stages (lower left panel of Fig. 9). Typically the Class 0 protostars have disks that contain about $1-10 \%$ of the total disk+envelope mass whereas the disks around the Class I sources contain 20-60\% of the disk+envelope mass. In both stages most of the derived disk masses are in the range of $\sim 0.01$ to $0.1 M_{\odot}$ with the median Class 0 disk mass of

\footnotetext{
2 Not to be confused with the infrared slope, $\alpha_{\mathrm{IR}}$, measured from the Spitzer data at 2.2 to $24 \mu \mathrm{m}$.
} 

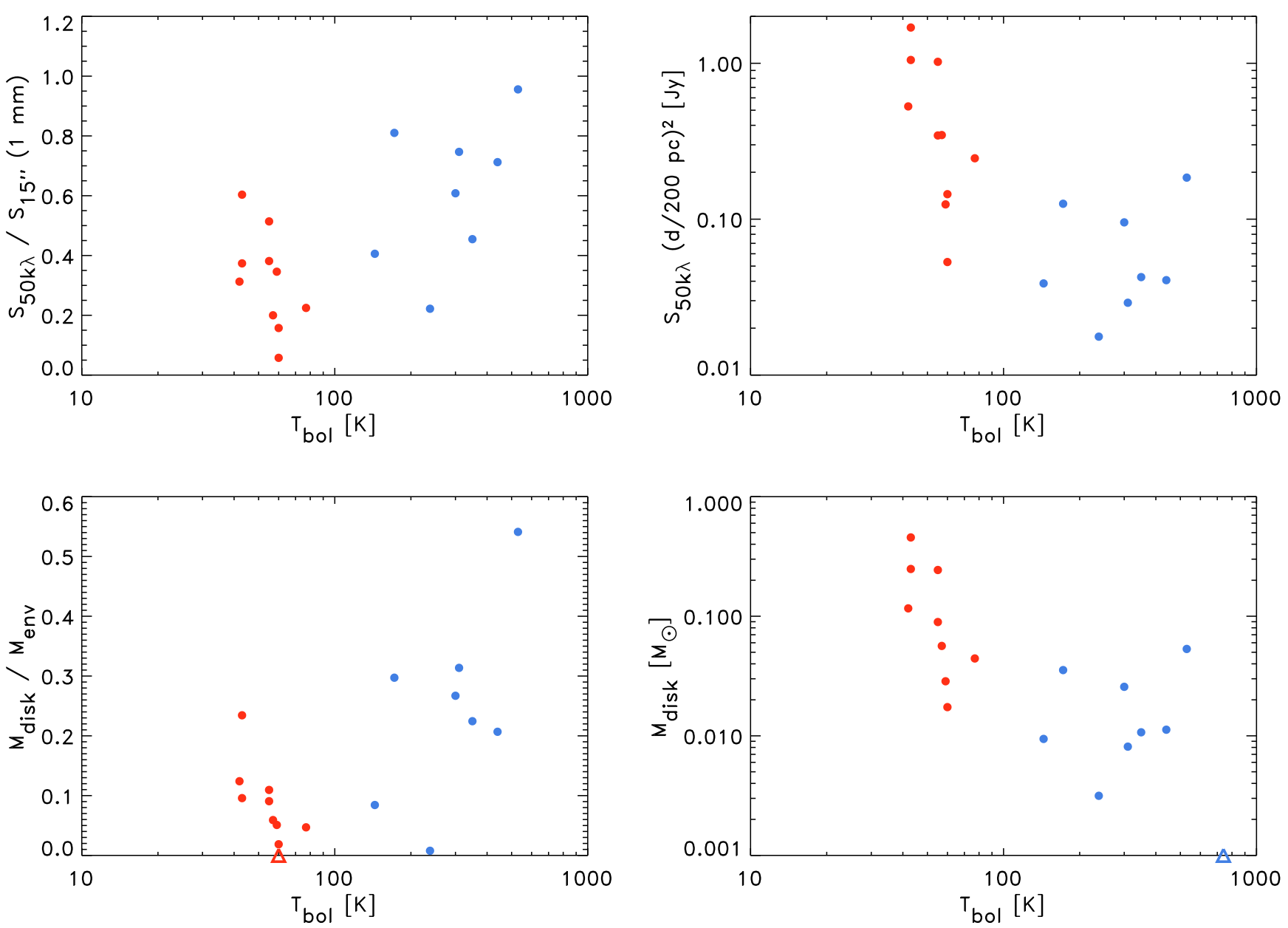

Fig. 9. Single-dish and interferometer fluxes as well as disk and envelope masses as function of source bolometric temperatures. The upper panels show the ratios of the interferometer and single-dish flux (left) and the interferometer flux (right) and the lower panels the ratio of the disk and envelope mass (left) and disk mass (right). Class 0 sources are shown with red symbols and Class I with blue symbols. In the lower left panel, L483 (Class 0 source with envelope-only emission; see discussion in text) is shown with the red triangle. In the lower right panel, IRS 54 (Class I source with no detected interferometric or single dish emission) is shown with a $3 \sigma$ upper limit to its disk mass $\left(0.001 M_{\odot}\right)$ from the SMA observations with the blue triangle.

$0.089 M_{\odot}$ and the median Class I disk mass of $0.011 M_{\odot}$. The drop in envelope masses from the Class 0 to I stages and the relatively low disk masses, suggests that a significant fraction of the mass of the central star is attained already during the most deeply embedded stages.

The results above do not show evidence for an increasing disk mass going from the Class 0 sources to the Class I sources in the observed samples. Thus, either the disks build up rapidly during the early, deeply embedded stages or their physical properties (e.g., grain properties, temperatures) are significantly different than those in more evolved stages. Andrews \& Williams (2005, 2007b) examined the relation between the disk masses for a large sample of Class I and II sources in Ophiuchus and Taurus and found evidence for a decrease of the disk masses by a factor 3 between these evolutionary stages from a median disk mass of $0.015 M_{\odot}$ to $0.005 M_{\odot}$.

Although most sources show compact emission, there are exceptions: the interferometric flux of the L483 Class 0 source is found to be consistent with envelope-only emission and does not require a central compact source. This is similar to the conclusion of Jørgensen (2004) who modeled $3 \mathrm{~mm}$ continuum emission of the source from OVRO observations. It is also in agreement with the measured spectral slope of the SMA continuum flux between 1.3 and $0.8 \mathrm{~mm}$ (Jørgensen et al. 2007a), which shows an index of $\approx 4$, consistent with optically thin dust in a larger scale envelope, contrasting the other Class 0 sources in the sample which have lower spectral indices in the range of 2-3 (Fig. 3 of Jørgensen et al. 2007a). It is puzzling that no direct evidence of a dust disk is seen for this source, given that it has a well-established protostellar outflow - which even seems to be in the process of actively dispersing the envelope. This may be a case, however, where we overestimate the contribution by the envelope on the longest baselines from the radiative transfer models. If the envelope, for example, has a larger flattened inner region or cavity, the envelope contribution on the longer baselines would be smaller and a central circumstellar disk required to explain the submillimeter emission. The mass of this disk would in that case be $\leqslant 0.01 M_{\odot}$ - derived from the long baseline interferometric data without taking the larger scale envelope contribution into account (Jørgensen et al. 2007a).

In a recent study Girart et al. (2009) modeled SMA continuum data for the Class 0 protostellar binary L723 and likewise found that a disk was not required to explain the compact continuum emission. Scaling their observed flux at $1.35 \mathrm{~mm}$ from 
Table 4. Envelope and disk masses for the full sample of Class 0 and I sources.

\begin{tabular}{llll}
\hline \hline Source & $\begin{array}{l}M_{\text {env }}{ }^{a} \\
{\left[M_{\odot}\right]}\end{array}$ & $\begin{array}{l}M_{\text {disk }}{ }^{b} \\
{\left[M_{\odot}\right]}\end{array}$ & $\begin{array}{l}M_{\text {star }}{ }^{c} \\
{\left[M_{\odot}\right]}\end{array}$ \\
\hline \multicolumn{4}{c}{ Class 0} \\
L1448-mm & 0.96 & 0.044 & $\ldots$ \\
IRAS2A & 1.0 & 0.056 & $\ldots$ \\
IRAS4A1 & $4.5^{d}$ & 0.46 & $\ldots$ \\
IRAS4A2 & $-^{d}$ & 0.25 & $\ldots$ \\
IRAS4B & $2.9^{d}$ & 0.24 & $\ldots$ \\
IRAS4B & $-^{d}$ & 0.089 & $\ldots$ \\
L1527 & 0.56 & 0.029 & $\ldots$ \\
L483 & 0.82 & $0.0^{e}$ & $\ldots$ \\
B335 & 0.93 & 0.017 & $\ldots$ \\
L1157 & 0.94 & 0.12 & $\ldots$ \\
& \multicolumn{2}{c}{ Class I } & \\
L1489-IRS & 0.11 & 0.018 & $1.3^{f}$ \\
TMR1 & 0.11 & 0.0094 & $\ldots$ \\
TMC1A & 0.12 & 0.035 & $0.35-0.7^{g}$ \\
GSS30-IRS3 & 0.096 & 0.026 & $\ldots$ \\
WL 12 & 0.054 & 0.011 & $\ldots$ \\
Elias 29 & 0.047 & 0.011 & $2.5^{h}$ \\
IRS 43 & 0.026 & 0.0081 & $1.0^{i}$ \\
IRS 54 & $\ldots$ & $<0.001$ & $\ldots$ \\
IRS 63 & 0.098 & 0.053 & $0.36^{h}$ \\
\hline
\end{tabular}

${ }^{a}$ Envelope mass determined from the direct scaling of the JCMT peak flux (minus the disk contribution). ${ }^{b}$ Disk mass assuming a uniform dust temperature of $30 \mathrm{~K} .{ }^{c}$ Central mass from Keplerian velocity profiles (see specific references for details). ${ }^{d}$ IRAS4A1 and IRAS4A2, respectively IRAS4B and IRAS4B' have larger scale circumbinary envelopes. $e^{e}$ The compact flux in L483 is consistent with being solely from the larger scale envelope. ${ }^{f}$ Stellar mass from Brinch et al. (2007), envelope and disk masses calculated under same assumptions as for the remainder of the sources using the continuum data from Brinch et al. ${ }^{g}$ Brown \& Chandler (1999). ${ }^{h}$ Lommen et al. (2008), ${ }^{i}$ This work. ${ }^{j}$ IRS 54 is undetected in both interferometric and single-dish maps. A $3 \sigma$ upper limit to its disk mass is estimated from the SMA observations.

the SMA at baselines of about $50 \mathrm{k} \lambda$ to our reference wavelength at $1.1 \mathrm{~mm}$ gives a flux of about $35 \mathrm{mJy}$ - or about $3-4 \%$ of the observed SCUBA peak flux of $1.1 \mathrm{Jy} \mathrm{beam}^{-1}$ from the JCMT/SCUBA legacy catalog (Di Francesco et al. 2008). Within our framework this fraction is exactly what is expected for envelope only contribution at these baselines, and in agreement with the interpretation by Girart et al. (2009) that no disk is required to explain the SMA continuum observations, but with a note that if the envelope would have an inner flattened region, an additional central dust component would be required - again with a typical mass $\lesssim$ a few $\times 0.01 M_{\odot}$.

\section{Analysis of line data: stellar masses}

In the previous section masses were determined for two of the three YSO components - their disks and envelopes. The resolved line observations obtained for the Class I sources provide information about the dynamical structure of their inner envelopes and disks and thereby a handle on the mass of the third component, the central star.

As mentioned above, significant differences exist between the components traced by the line observations for the different sources in the sample. Figure 10 compares the near-infrared $1.6 \mu \mathrm{m}$ images to the $\mathrm{HCO}^{+}$emission in TMR1, TMC1A,
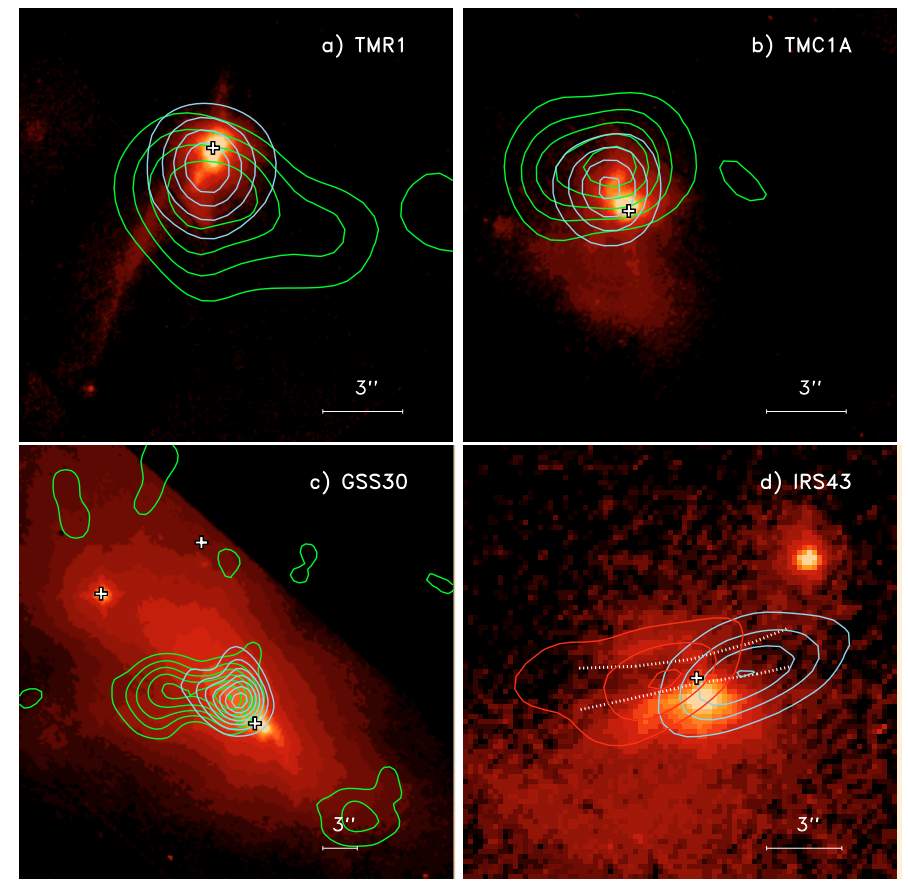

Fig. 10. Archive $1.6 \mu \mathrm{m}$ HST/NICMOS images of TMR1, TMC1A, GSS30 and IRS 43 (see Terebey et al. 1998; and Allen et al. 2002, for details about the data) with $\mathrm{HCO}^{+} 3-2$ emission contours from the SMA overlaid. Panel a)-c) show TMR1, TMC1A and GSS30 with blue and green contours corresponding to blue-shifted emission integrated from $V_{\mathrm{LSR}}-2 \Delta V$ to $V_{\mathrm{LSR}}-\Delta V$ and $V_{\mathrm{LSR}}-\Delta V$ to $V_{\mathrm{LSR}}$ with $\Delta V=1.0 \mathrm{~km} \mathrm{~s}^{-1}$ (TMR1 and TMC1A) and $\Delta V=1.5 \mathrm{~km} \mathrm{~s}^{-1}$ (GSS30). For IRS 43 in panel $\mathrm{d}$ the blue and red contours correspond to blue- and red-shifted emission integrated from $V_{\mathrm{LSR}}-2 \Delta V$ to $V_{\mathrm{LSR}}-\Delta V$ and $V_{\mathrm{LSR}}+\Delta V$ to $V_{\text {LSR }}+2 \Delta V$ with $\Delta V=1.5 \mathrm{~km} \mathrm{~s}^{-1}$. In that panel the white dotted lines indicate the rough extent of the dark lane in the HST image.

GSS30-IRS1 and IRS 43. The first three of these sources show a clear alignment with the near-infrared scattering nebulosities whereas the fourth, IRS 43, shows the $\mathrm{HCO}^{+}$(and also $\mathrm{HCN}$ ) emission aligned with the dark lane across the source. Likewise, in the first order moment (velocity) maps (Fig. 11), TMR1, TMC1A and GSS30 show significantly different morphologies from IRS 43 and IRS 63: the former sources show evidence of blue-shifted emission over most of their maps, with velocities decreasing toward the systemic velocities at the largest distances. In contrast IRS 43, in particular, shows a clear large-scale velocity gradient relative to the continuum peak position and systemic velocity, potentially indicative of rotation as also inferred for IRS 63, Elias 29 (Lommen et al. 2008) and L1489-IRS (Brinch et al. 2007). In the following we focus on the use of $\mathrm{HCO}^{+}$as a tracer of rotation in these sources and include a small discussion of the sources showing outflow cavities and the limitations of $\mathrm{HCO}^{+}$as a tracer in Appendix A. Some details about the individual Class I sources are given in Appendix B.

\subsection{Keplerian rotation and stellar mass in IRS 43}

Four sources in our sample, IRS 43, IRS 63 and Elias 29 (Fig. 5) as well as L1489-IRS (Brinch et al. 2007), show elongated $\mathrm{HCO}^{+}$emission stretching across the location of the YSO. As discussed by Brinch et al. (2007) and Lommen et al. (2008) the velocity gradients toward L1489-IRS, IRS 63 and Elias 29 can be interpreted as having their origin in the inner 

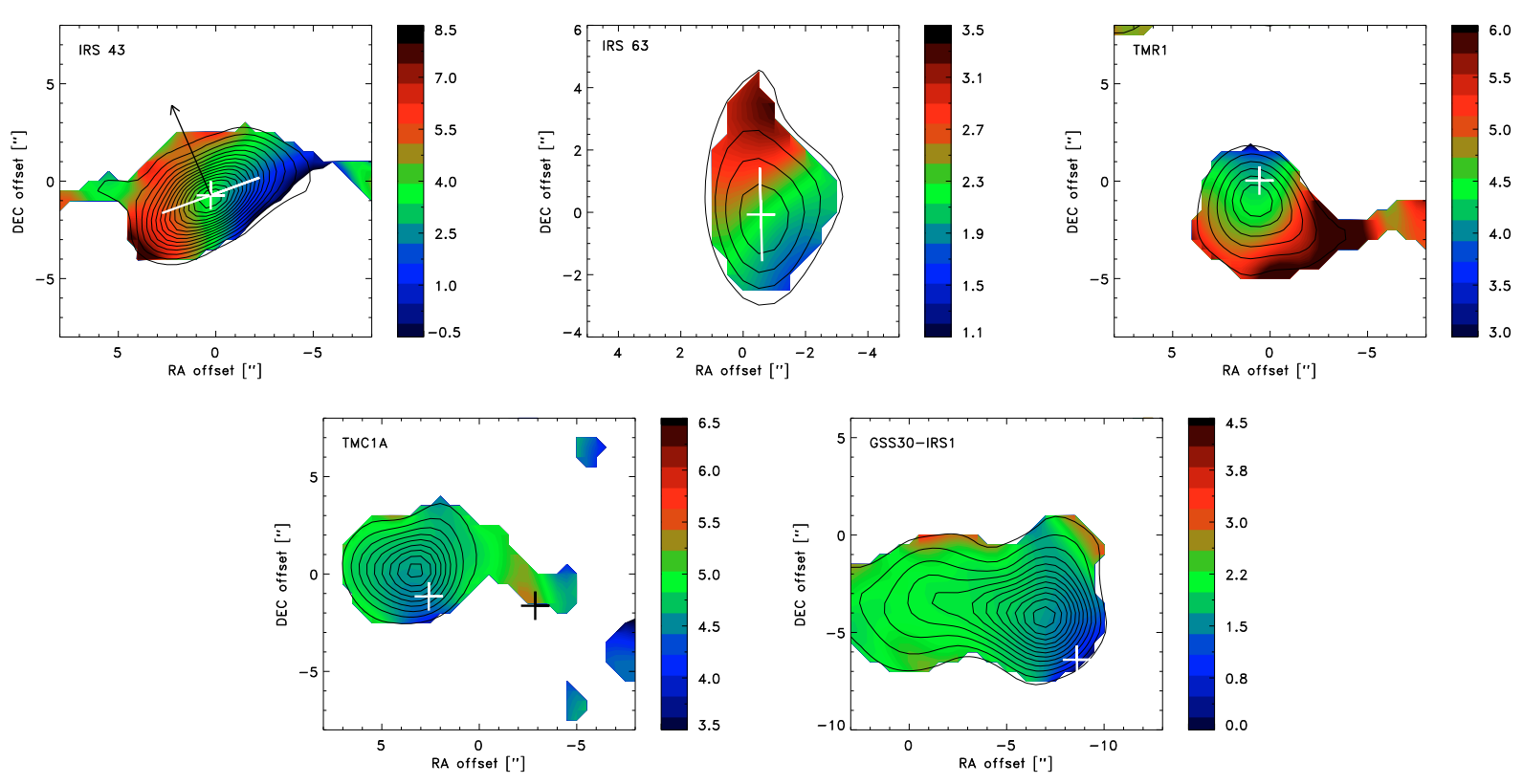

Fig. 11. Moment one (velocity) maps of the $\mathrm{HCO}^{+}$emission for IRS 43, IRS 63, TMR1, TMC1A and GSS30-IRS1. In all panels the plus-signs indicate the location of the continuum positions (for GSS30-IRS1 the near-/mid-infrared source). In the IRS 43 and IRS 63 panels the white line shows the direction of the continuum structure. In the IRS 43 panel, the black arrow furthermore shows the direction of the embedded near-IR Herbig-Haro objects (Grosso et al. 2001) and thermal jet (Girart et al. 2000).

envelope/circumstellar disk around each source. A number of effects point toward this also being the case for IRS 43:

1. both $\mathrm{HCN}$ and $\mathrm{HCO}^{+}$show an elongated structure with a large scale velocity gradient around the continuum peak/systemic velocity;

2. a Gaussian fit to the continuum data in the $(u, v)$ plane shows a structure which is elongated in the same direction with a position angle of $-70^{\circ}$ (measured from north toward east): its deconvolved major axis is about $2^{\prime \prime}$ (280 AU) and its minor axis about a tenth of an arcsecond, consistent with emission from a disk seen at a high inclination angle, close to edge-on.

3. The direction of the Herbig-Haro objects (Grosso et al. 2001) and proposed radio thermal jet (Girart et al. 2000) are with a position angle of $20-25^{\circ}$, perpendicular to this extended structure (Figs. 11 and B.1).

The narrow continuum structure and the alignment with the $\mathrm{HCO}^{+} 3-2$ emission is also seen in the IRS 63 data from Lommen et al. (2008). For that source, the velocity field could be well-fit with a Keplerian profile around a $0.37 M_{\odot}$ central object for an inclination of $30^{\circ}$ (as hinted by modeling of the spectral energy distribution of the source). A position-velocity plot of the $\mathrm{HCO}^{+} 3-2$ emission toward IRS 43 is shown in Fig. 12. The position velocity diagram shown here has been extracted along the major axis of the $\mathrm{HCO}^{+}$emission at a position angle of $-70^{\circ}$. The velocity profile is consistent with Keplerian rotation.

The extent of the $\mathrm{HCO}^{+}$emission $\left(\approx 15^{\prime \prime}\right)$ suggests that its origin is not purely in the central disk, but also the inner regions of the rotating envelope ( $r \lesssim 1000 \mathrm{AU})$. This is further supported by the comparison between the single-dish spectrum and a spectrum extracted from the interferometric data integrated over the extent of the JCMT single-dish beam (Fig. 13). About $40 \%$ of the single-dish flux is recovered by the interferometer, suggesting the presence of some extended emission resolved out by the interferometer - although this fraction is significantly less than typically seen, e.g., in the more deeply embedded Class 0 protostars (e.g., Jørgensen et al. 2007a).
To derive the mass of the central object a position-velocity curve is extracted by fitting the position of the peak emission for each channel in the $(u, v)$-plane at baselines larger than $20 \mathrm{k} \lambda$ and projecting this on the major axis of the $\mathrm{HCO}^{+}$emission (right panel, Fig. 12). To this curve, a $\chi^{2}$-fit is performed with a straightforward Keplerian rotation curve with two free parameters, the systemic velocity and central mass. A best fit is obtained for a central mass of $1.0 M_{\odot}\left(2 \sigma\right.$ confidence levels of $\left.\pm 0.2 M_{\odot}\right)$ and a systemic velocity of $4.1 \mathrm{~km} \mathrm{~s}^{-1}$. This mass estimate is of the "enclosed" mass within the resolution of the interferometer, but since it is significantly higher than the best estimate of the envelope and disk masses of 0.026 and $0.0081 M_{\odot}$ respectively (see Sect. 4), it is likely close to the total mass of the central star. This is also a lower limit since it is not corrected for inclination, but given the small minor axis relative to the major axis from the Gaussian fit to continuum emission, it is not unreasonable to assign a high inclination angle corresponding to an edge-on disk.

In summary, we detect signatures of Keplerian rotation in the $\mathrm{HCO}^{+} 3-2$ emission for 4 Class I sources allowing direct determinations of their central stellar masses, which range from about 0.3 to $2.5 M_{\odot}$. As discussed in Appendix A.2, the $100 \mathrm{AU}$ scales of embedded protostars can only be traced with $\mathrm{HCO}^{+}$ 3-2 in sources with envelope masses less than about $0.1 M_{\odot}$, however. Future high sensitivity ALMA observations will be required to observe more optically thin isotopes, to determine the dynamical structure of the more deeply protostars.

\section{Discussion}

\subsection{Effects of assumed dust properties and temperatures on disk masses}

As in most other studies in the literature, two assumptions in our study are that the disk can be characterized by a uniform temperature and by a single unchanging dust opacity law throughout its extent and evolution. Both these assumptions may affect the 

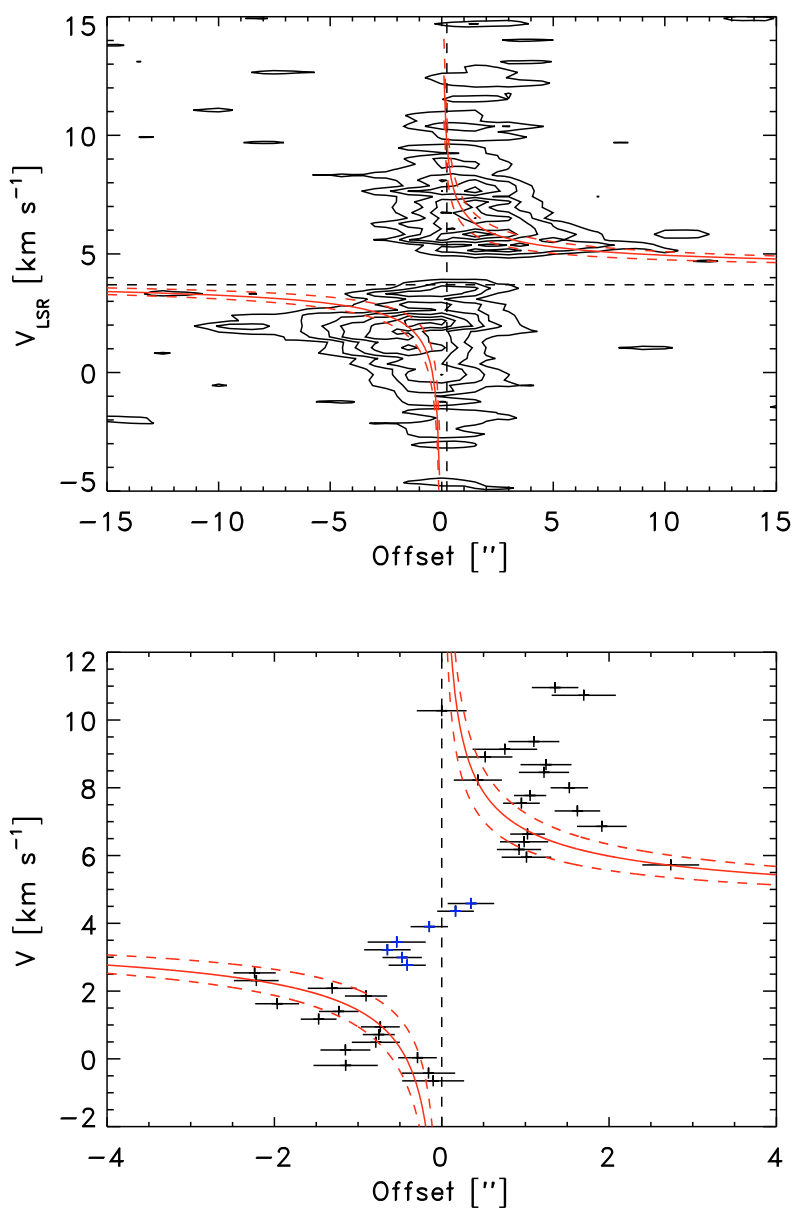

Fig. 12. Position-velocity (PV) diagram for the $\mathrm{HCO}^{+}$emission in IRS 43 directly from the image-plane (upper panel) and from fitting the individual channels in the $(u, v)$-plane (lower panel). The PV diagram has been measured along the major axis of the $\mathrm{HCO}^{+}$emission at a position angle of $-70^{\circ}$ (measured from north toward east). Overplotted are the predicted curves for Keplerian rotation around a $1.0 M_{\odot}$ central object (solid) line (best fit to $(u, v)$-data), which can be compared to the dashed lines showing the same predictions for 0.6 and $1.4 M_{\odot}$ central objects.

systematic trends observed in the data: for the temperature an increase in luminosity increases the mass-weighted temperature of the disk, while the shielding by the disk itself will lead to a lower mass weighted temperature with increasing mass. As an example, Fig. 14 compares the temporal evolution of the temperature at a radius of $200 \mathrm{AU}$ between rays through the model with different inclinations with respect to the disk plane in the simulation of Visser et al. (2009). Also shown is the evolution of the luminosity in the simulations. Early in the evolution, before the disk is formed, the temperature tracks the luminosity changes closely, but as the disk forms (at about $10^{5}$ years) the temperature at inclinations directed through the disk drops sharply, even though the luminosity continues to increase. The strong increase in total luminosity at about $3 \times 10^{5}$ years is directly reflected in the temperature profiles.

Figure 15 compares the relationships between (sub)millimeter flux and disk mass adopted by Terebey et al. (1993), Andrews \& Williams (2007b) and Jørgensen et al. (2007a) to the relationship predicted by the radiative transfer modeling of the collapse simulation from Visser et al. (2009): it is clearly seen that the models require a steeper relationship between the

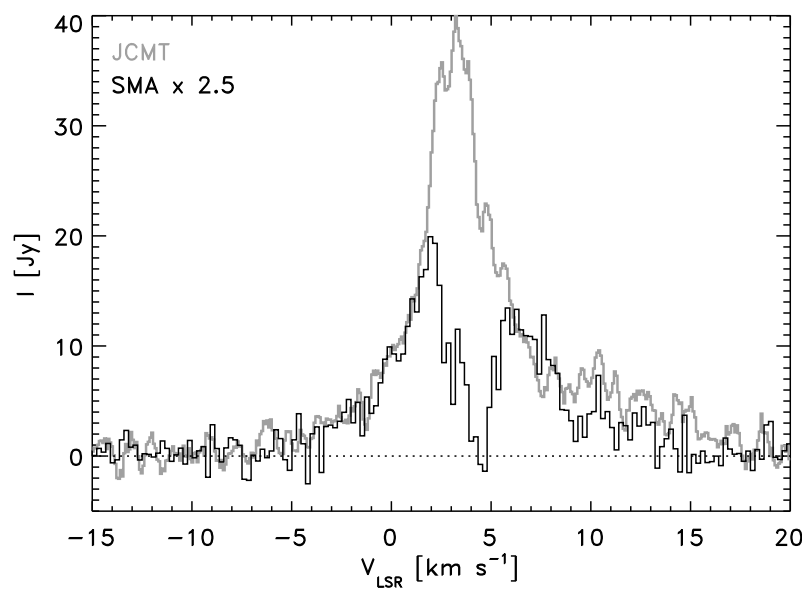

Fig. 13. IRS 43: comparison between $\mathrm{HCO}^{+} 3-2$ spectrum from the SMA data integrated over the single-dish beam and JCMT observations. The interferometric spectrum has been scaled by a factor 2.5 .

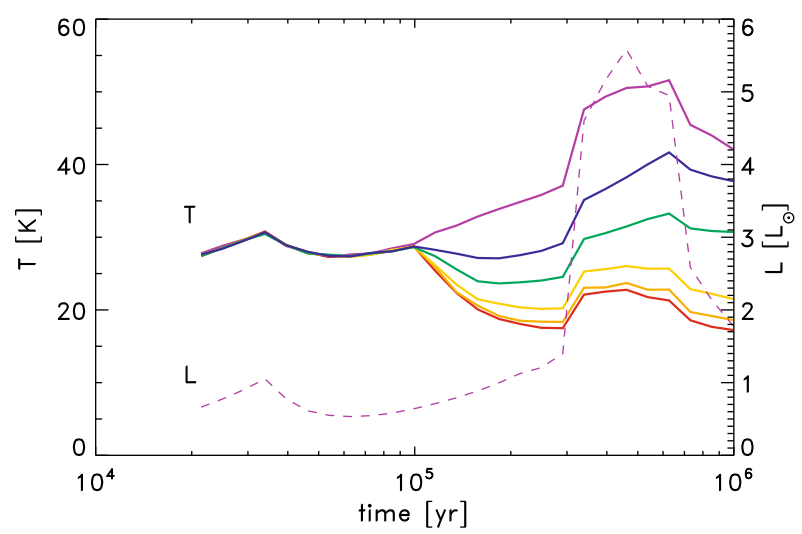

Fig. 14. Temporal evolution of the temperature and luminosity in the simulation of Visser et al. (2009). The solid lines shows the temperature at $200 \mathrm{AU}$ along rays inclined with $0,5,10,15,20$ and $90^{\circ}$ relative to the disk plane (from bottom to top; i.e., the line with the highest temperature corresponds to an inclination of $90^{\circ}$ - perpendicular to the disk plane). The dashed line shows the corresponding evolution of the luminosity.

submillimeter flux and disk mass than what is obtained with a single constant temperature: to calculate the disk mass from the submillimeter flux in the model, one would need a temperature scaling as:

$T=11.3 \mathrm{~K}\left(\frac{M_{\mathrm{disk}}}{0.01 M_{\odot}}\right)^{-0.25}\left(\frac{L_{\star}}{L_{\odot}}\right)^{0.1}$

as shown with the solid line in Fig. 15 - i.e., an increasing temperature with increasing luminosity (and thus heating) and a decreasing temperature with increasing disk mass (and thus shielding at a given radius). Although the variations in the temperature with disk mass and stellar luminosity are slow, they still introduce a systematic gradient in the derived disk masses by up to a factor of 4 through the evolution of the embedded stages of the YSOs. It thus appears that the disk masses in the later stages relative to the early stages can be underestimated by about this factor - solely due to the evolution of the protostellar system. This may explain the slightly higher masses for the Class 0 sources in Fig. 9. 


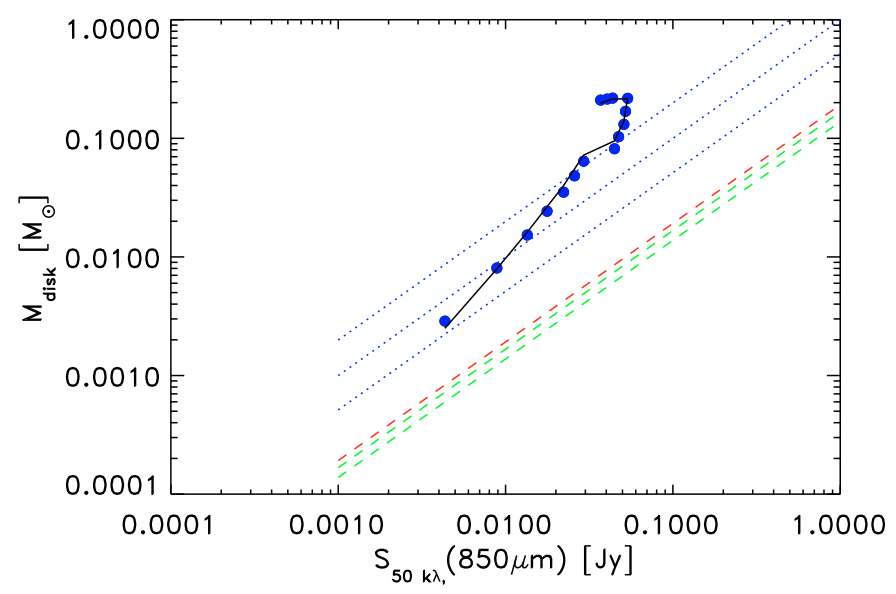

Fig. 15. Relationship between disk mass and $850 \mu \mathrm{m}$ interferometer flux assuming a distance of $250 \mathrm{pc}$ : the three lower straight lines indicate the linear relationships from Terebey et al. (1993) (lowest), Andrews \& Williams (2007b) (middle) and Jørgensen et al. (2007a) (top) scaled to the same distance and wavelength. The filled symbols indicate the relationship found for the radiative transfer modeling of the Visser et al. (2009) collapse simulation discussed in the text. The solid line through these points indicates a relationship where the temperature used to relate the disk mass and flux follows Eq. (4). Finally, the three dotted lines indicate predicted linear relationships using the dust properties from the simulations of Visser et al. (2009) with temperatures of 8, 11 and $16 \mathrm{~K}$ (from top to bottom).

Figure 16 compares the derived disk masses to bolometric temperature as well as the ratio of the source luminosity over the envelope mass, $L_{\star} / M_{\text {env }}$. The latter ratio is an alternative evolutionary tracer, increasing with time as long as the YSO luminosity is dominated by accretion and the stellar mass (and luminosity) increases, whereas the envelope dissipates and its mass decreases. In this figure the Class 0 disk masses are decreased by a factor of 2 and the Class I disk masses increased by a factor of 2 to simulate the temperature effect discussed above. With this correction, the Class 0 and I disk masses are roughly constant with a median mass of about $0.04 M_{\odot}$ for the full sample.

The assumption of a constant unchanging dust opacity law similar to the large-scale envelope dust may also be wrong: in particular, grain growth may increase the dust opacity at submillimeter wavelengths leading to a lower derived disk mass for a given interferometric flux. That something like this may be going on is hinted by the observation that the compact components around the Class 0 sources show spectral slopes, $\alpha$, of the emission $F_{v} \propto v^{\alpha}$, at 230 and $345 \mathrm{GHz}$ in the range 2-3 (Jørgensen et al. 2007a). This suggests that the disks are either optically thick or have significantly different dust opacities than standard ISM dust. As discussed in Jørgensen et al., the disk masses implied for the Class 0 objects suggest that only the most massive ones may be marginally optically thick. Resolved observations at multiple wavelengths and measurements of their sizes are required to address this. On the other hand, the low spectral indices confirm that the compact components around the Class 0 sources are not associated with the larger scale envelopes for which an $\alpha$ of 3.5-4 is expected.

Other dust opacity laws or temperatures have been used in literature: Andrews \& Williams (2007b) adopted an empirical scaling based on modeling of the disk SEDs, corresponding to an equivalent dust temperature of $20 \mathrm{~K}$ and a dust opacitylaw following Beckwith et al. (1990) normalized to the dust opacity at $1000 \mathrm{GHz}$ of $0.1 \mathrm{~cm}^{2} \mathrm{~g}^{-1}$ (for a dust-to-gas ratio
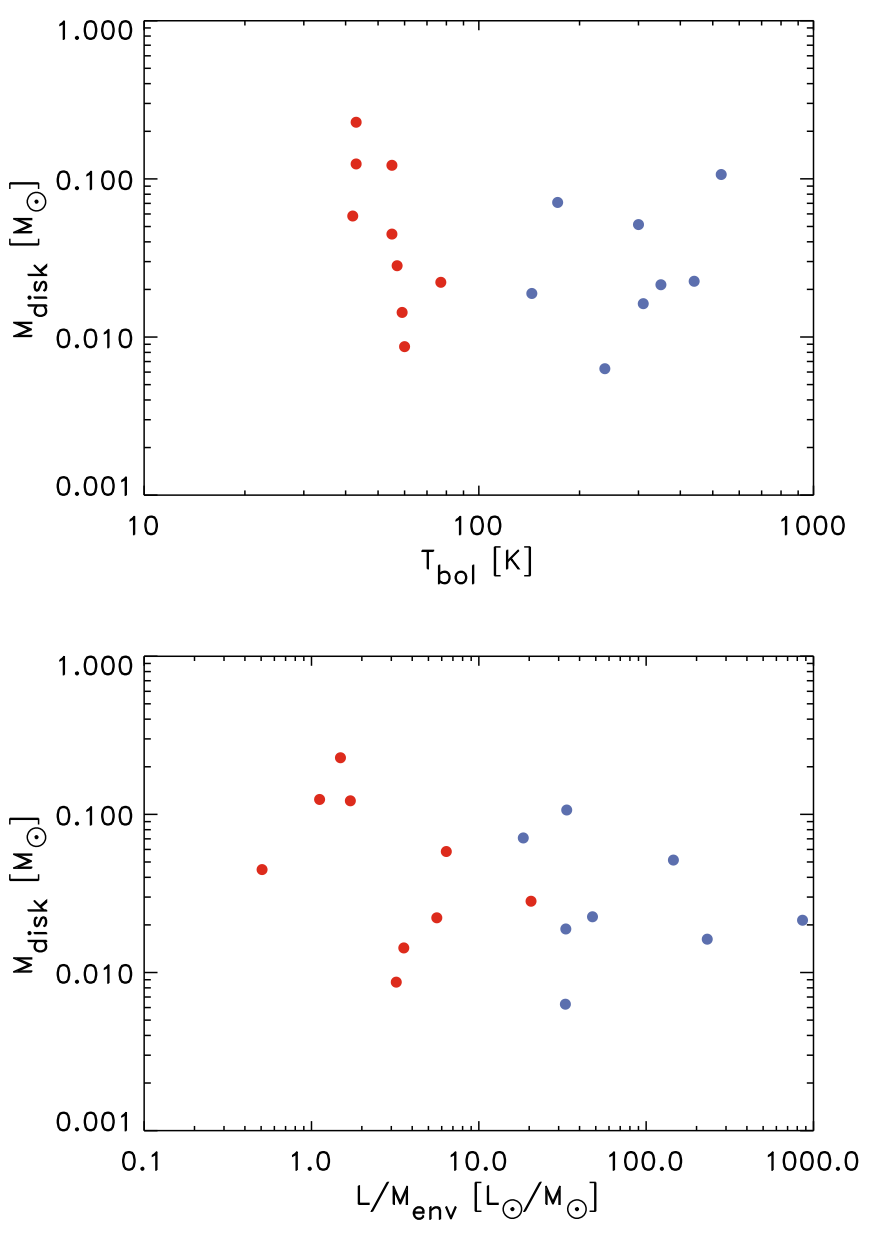

Fig. 16. Disk masses as function of bolometric temperature (upper) and source luminosity/envelope mass ratio (lower). In comparison to Fig. 9, the Class 0 disk masses have been decreased by a factor 2 and the Class I disk masses increased by a similar amount - simulating the evolutionary effect in the relationship between flux and disk mass suggested by Fig. 15.

of 1:100). The lower temperature leads to higher disk masses for a given $1.1 \mathrm{~mm}$ flux by a factor of 2.3 whereas their adopted dust opacity-law corresponds to lower disk masses by a factor 2.0 compared to ours. Likewise, Terebey et al. (1993) adopted a dust temperature of $40 \mathrm{~K}$ and adopted a dust opacity law with a mass opacity at $250 \mu \mathrm{m}$ of $0.1 \mathrm{~cm}^{2} \mathrm{~g}^{-1}$. If compared at a common wavelength of $850 \mu \mathrm{m}$, these three choices of parameters result in disk masses that agree within $\pm 15 \%$ from the middle value from Andrews \& Williams (2007b).

However, systematic changes in the dust properties occurring during the evolution of the young protostars could have an important effect on our derived disk masses and observed trends. For example, the dust opacities at $500 \mu \mathrm{m}-1 \mathrm{~mm}$ from Ossenkopf \& Henning (1994) increase by as much as an order of magnitude depending on the density at which the grains have coagulated, although the effect is significantly smaller for grains with ice mantles. As shown by D'Alessio et al. (2001) grain growth increases the dust opacity at a specific wavelength, $\lambda$, as long as the largest grains are smaller than $\lambda$. In a scenario where grains grow toward sizes inferred for $\mathrm{T}$ Tauri disks ( $\sim 1 \mathrm{~mm}$; e.g., D’Alessio et al. 2001) as the protostellar system evolve through its embedded stages the derived disk masses for the more evolved sources would be overestimated under our 
assumptions of an unchanging dust opacity law. This would work in the opposite direction of the temperature effect - toward even more massive disks in the Class 0 compared to Class I stages. It cannot be ruled out that grain growth would appear in a fashion not straightforwardly increasing with "age" of the studied young stellar objects, e.g., if the observed dust in the Class 0 sources is located in regions with relatively higher densities for a significant fraction of time. Based on the current observational data, it is not possible to separate these scenarios, however: further resolved multi-wavelength images of the dust continuum emission from the envelopes and disks around protostars or of well-calibrated gas tracers (taking their chemistry into account) are required.

\subsection{Collapse and disk formation models}

Power-law density profiles are simplified parameterizations of the underlying envelope density profile and the envelopes are not spherical due to, e.g., rotation and the action of protostellar outflows.

In the framework of the Shu (1977) and Terebey et al. (1984) models for inside-out collapsing envelopes, the density profile flattens from a stationary $r^{-2}$ density profile through a freefalling $r^{-1.5}$ profile to a flattened inner envelope where the angular momentum roughly balances gravity. Terebey et al. (1993) examined the millimeter emission from such envelope models using analytical descriptions for the envelope density and temperature profiles and used those to compare IRAM $30 \mathrm{~m}$ singledish continuum observations (11"; at $1.3 \mathrm{~mm}$ ) to OVRO interferometric observations (4-10"; at $2.7 \mathrm{~mm}$ ). Consistent with Fig. 6 they found that the emission in the interferometric observations is completely dominated by a central compact component whereas most of the observed single-dish flux in fact has its origin in the collapsing envelope. The dominance of the disk to the interferometric flux was found by Terebey et al. to be due to (i) the smaller beam of the interferometric data and negligible envelope mass on those scales and; (ii) the steeper wavelength dependence found for the dust emission in the optically thin envelope.

We expand this analysis by introducing the formation of a circumstellar viscous disk in the center using the models of Visser et al. (2009) and following the evolution of the submillimeter brightness profiles observed by an interferometer: Visser et al. adopted the framework of Cassen \& Moosman (1981) and Terebey et al. (1984) to describe a collapsing envelope and introduced the formation and growth of a 2-dimensional central disk and star similar to, e.g., Yorke \& Bodenheimer (1999). The models of Visser et al. also include the presence of an outflow cavity with a growing opening angle through the evolution consistent with observations (e.g., Arce \& Sargent 2006). Using the axisymmetric radiative transfer code "RADMC" (Dullemond \& Dominik 2004), Visser et al. calculated the temperature profile for time-steps in the above model description and furthermore followed the chemical history of particles during the collapse and disk formation. For this purpose, we adopt the temperature and density profiles of the envelope (with outflow cavity) and disk from Visser et al., as well as their prescription for the evolution of the stellar luminosity and effective temperature and dust parameters, and ray-trace these. Figure 17 shows the evolution of the predicted visibility curves at different time-steps for two representative models from the grid of Visser et al.

Both panels clearly illustrate the effect discussed above: until the formation of the circumstellar disk more than $95 \%$ of the total flux at a given wavelength is emitted on baselines shorter
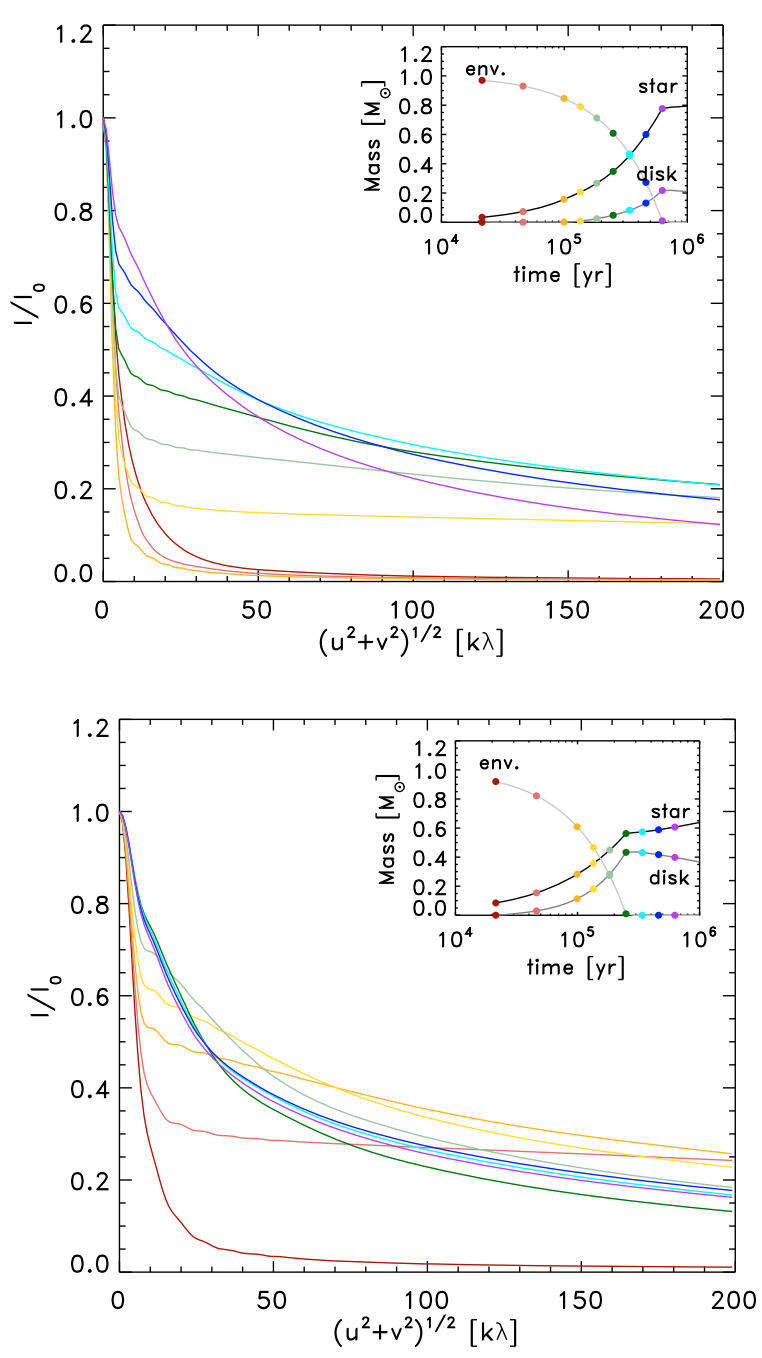

Fig. 17. Evolution of the predicted visibility amplitude curves (normalized by the zero-spacing flux) as function of projected baseline lengths for two models for envelope collapse and disk formation from Visser et al. (2009) characterized by the solid-body rotation rate, $\Omega_{0}$, and the effective sound speed $c_{s}$. Upper: $\Omega_{0}=10^{-14} \mathrm{~s}^{-1}$ and $c_{\mathrm{s}}=0.19 \mathrm{~km} \mathrm{~s}^{-1}$. Lower: $\Omega_{0}=10^{-13} \mathrm{~s}^{-1}$ and $c_{\mathrm{s}}=0.26 \mathrm{~km} \mathrm{~s}^{-1}$. The different curves correspond to snapshots at different times indicated in the upper right insert in each panel, also showing the evolution of the envelope, disk and stellar masses.

than $50 \mathrm{k} \lambda$-corresponding to a ratio between the $1.1 \mathrm{~mm}$ interferometric flux and the peak $850 \mu \mathrm{m}$ single-dish flux of less than a few $\%$ (depending on the exact spectral slope between the observations at these differing wavelengths) in accordance with the results above. Once the disk is formed, the longer baseline flux rapidly increases - first simultaneously on all baselines and then more rapidly on the shorter baselines as the disk grows to sizes large enough to be resolved by the interferometric observations. It should be noted that even though the outflow cavity is opened up early in the evolution of the collapsing protostar, it does not provide significant compact emission, e.g., due to heated material in the outflow cavity walls, to affect the evolution of the observed interferometric flux.

An alternative explanation to the disk hypothesis favored in this work is that there is an enhancement of the density in the envelope profile at small scales. As an example, Chiang et al. (2008) adopted the theoretical collapse models of Tassis \& Mouschovias (2005) and compared those to $2.7 \mathrm{~mm}$ 
interferometric data from Looney et al. (2000). The models of Tassis \& Mouschovias (2005) simulate the formation of a protostellar object from a cloud core in the presence of magnetic fields. In their simulation, ambipolar diffusion leads to the formation of a thermally and magnetically super-critical core which contracts dynamically. Initially this core has a density profile $n \propto r^{-1.7}$, but as mass and magnetic flux is accreted from the envelope onto the central protostar, a region of enhanced magnetic fields is formed driving an outward-propagating shock and causing a density enhancement growing quasi-periodically from scales of $\sim 100 \mathrm{AU}$. This density enhancement on small scales will result in a compact component observable by the interferometer which is slightly resolved on long baselines and thereby in some cases alleviates the need for a circumstellar disk on those scales as shown by Chiang et al. (2008).

Still, the conclusions of both studies are similar: a density enhancement is required in the collapsing envelope on small scales - above what would be expected from, e.g., a free-falling envelope. The question is whether this component is inherent to the collapse profile or the first sign of the formation of a circumstellar disk. A straightforward, although currently observationally challenging, way to address this issue is through resolved line observations of the innermost envelope: the resolved line observations of the Class I sources in this sample (Brinch et al. 2007; Lommen et al. 2008) show clear signatures of Keplerian rotation, which argues in favor of the disk scenario for the sources in those evolutionary stages at least. For the more deeply embedded protostars future ALMA observations are needed to confirm the dynamical nature of the central compact dust component.

\subsection{Constraints on evolutionary models}

The presented observations and methodology make it possible to directly constrain models for the evolution of young stellar objects - in particular, concerning the transport of matter from the larger scale envelope to the central star. This is in principle also the most direct way for estimating the evolutionary status of individual young stellar objects - rather than indirect indicators such as their spectral energy distributions or similar. Figure 18 compares the predicted stellar and disk masses relative to the envelope masses (i.e., $M_{\text {star }} / M_{\text {env }}$ vs. $M_{\text {disk }} / M_{\text {env }}$ ) from the models of Visser et al. (2009) to our observed values. The thickened part of the lines in this plot indicates where the Class 0 and I sources would fall on the evolutionary track given their measured $M_{\text {disk }} / M_{\text {env }}$ ratios.

For the observed ratios of $M_{\text {disk }} / M_{\text {env }}$ for the Class 0 sources, the models predict ratios of stellar to envelope masses $M_{\mathrm{star}} / M_{\mathrm{env}}$ in the range $\approx 0.2-0.6$, i.e., systems that have accreted $\approx 15-40 \%$ of their final mass - in accordance with the phenomenological definition of objects in the Class 0 stage. Likewise the Class I sources are predicted to have more massive stars than envelopes - again, in accordance with the phenomenological distinction between Class 0 and I objects. For the Class I sources with measured stellar masses, the observed disk-to-stellar mass ratios are in the $1-10 \%$ range, whereas the models predict a higher ratio of $20-25 \%$ in the late Class I stage (i.e., overestimate the disk mass relative to the stellar mass). It is seen that the predicted stellar masses for the Class 0 sources with this model are a few $\times 0.1 M_{\odot}$.

The low disk masses relative to stellar masses suggest that material is quickly and efficiently accreted from the envelope through the disk onto the star. This is also directly illustrated by the comparison between the observed and modeled stellarto-disk mass ratios for the five Class I sources for which both have been determined. For the Class I sources the observed disk masses are $1-10 \%$ of the corresponding stellar masses whereas the models predict ratios of $20-25 \%$. This suggests that the material is processed more quickly through the circumstellar disks than what is given by the models. Looney et al. (2003) suggested that this was the case based on their conclusion that Class 0 protostars have disks that are not more massive than their Class I counterparts. Our results are in agreement with this general conclusion.

It is worth emphasizing though, that our analysis does find evidence for disks around the Class 0 sources. If the Class 0 sources indeed are precursors to the Class I sources in this sample, this in turn would suggest that circumstellar disks are formed early in the evolution of low-mass protostars. Without resolved line observations it is not possible to address whether these disks in fact are rotationally supported Keplerian disks. The absence of a clear rotation signature in the disk around NGC 1333-IRAS2A (Brinch et al. 2009) combined with its large apparent size from the dust continuum measurements (Jørgensen et al. 2005a) could perhaps be consistent with it being an unstable "pseudo-disk" as in the framework of Galli \& Shu (1993a,b) - although it may also simply be that the evolutionary time-scale for IRAS2A still has been too short for the disk to establish Keplerian rotation (Brinch et al. 2009).

An open question in the comparison to the theoretical models is how important outflows are in carrying away envelope material. The observed increasing opening angles of outflows with increasing age of the protostar has been interpreted as evidence for the erosion of the protostellar envelope by the outflow (Velusamy \& Langer 1998; Arce \& Sargent 2006) in part explaining the observed shift in the peak between the core and initial mass function (e.g., Myers 2008). In the models of Visser et al. (2009) and shown in Fig. 18 the amount of material carried away by the outflow only amounts to a few percent. Generally, a more efficient outflow dispersal of the core would decrease the disk and stellar masses relative to the envelope masses - corresponding to a diagonal shift of a given modeled object in Fig. 18 - roughly along the evolutionary tracks. Therefore, unless the outflow preferentially alter the accretion onto the disk relative to the star, the ratio between the masses of the two will not be changed and thus also not the implications discussed here.

A final interesting possibility is that the flat distribution of disk masses from the Class 0 to I stages reflects the stability of the disks, given the suggestion that the luminosity problem of low-mass protostars can be explained if mass is accreted on large scales in the disks and from there onto the central star in highly episodic events: in their models for collapse of a dense core and formation of a protostar and disk, Vorobyov \& Basu (2006) find strong episodic accretion once the disk has formed which keeps its mass lower than the stellar mass at all points during the protostellar evolution. Given that the recent Spitzer/c2d results suggest that the luminosity problem extends to the earliest Class 0 stages (Evans et al. 2009), it is tempting to suggest that material already at these stages indeed ends up in circumstellar disks and from there is accreted toward the central star in bursts when a local instability criterion is exceeded - e.g., related to comparison between the disk size/mass and stellar mass. This could in turn flatten the distribution of disk masses as function of bolometric temperatures, with an upper envelope of disk masses of $0.05-0.1 M_{\odot}$ corresponding to the maximum values observed for our sample of Class 0 and I protostars. It should be emphasized that since our sample is rather small, biases may have been introduced in its selection. Future studies of larger samples of protostars with current and upcoming (sub)millimeter interferometers will be needed to address this potential bias. 


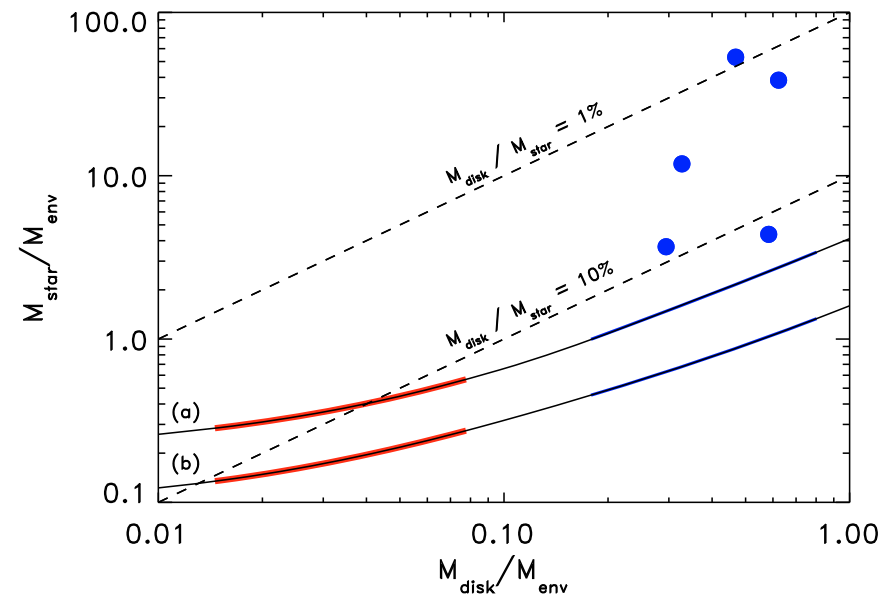

Fig. 18. Predicted stellar mass, $M_{\text {star }}$, vs. disk mass, $M_{\text {disk}}$, both measured relative to the envelope mass $M_{\text {env }}$ in the models of Visser et al. (2009) with $\Omega_{0}=10^{-14} \mathrm{~s}^{-1}$ and $c_{s}$ of a) $0.19 \mathrm{~km} \mathrm{~s}^{-1}$ and b) $0.26 \mathrm{~km} \mathrm{~s}^{-1}$ (solid lines; see also Fig. 17). On this model track, the thickened lines indicate where the Class 0 and I objects would be located given their distribution of measured $M_{\text {disk }} / M_{\text {env }}$ values (red and blue, respectively). The sources for which stellar, disk and envelope masses are measured are shown with symbols. Finally, the dashed lines indicate $M_{\text {disk }} / M_{\text {star }}$ ratios of $1 \%$ (upper) and $10 \%$ (lower).

\section{Summary and outlook}

We have presented a comprehensive analysis of the dust continuum envelope emission of a sample of 20 embedded low-mass YSOs (Class 0 and I) based on submillimeter interferometric observations from the Submillimeter Array probing scales of a few hundred $\mathrm{AU}$ and single-dish continuum observations from the JCMT (scales larger than $2000 \mathrm{AU}$ ). In addition we discuss $\mathrm{HCO}^{+} 3-2$ line emission for the Class I sources in the sample. With these data we have characterized the evolution of the three main components YSOs - the envelope, disk and stellar masses - and the relation between these compared to theoretical models. The main conclusions are as follows:

1. The millimeter dust continuum emission from both the interferometric observations (on scales of 250-500 AU) and single-dish observations (scales larger than 2000 AU) decreases in strength from the more deeply embedded Class 0 to the more evolved Class I sources. Relative to the singledish data, the interferometric continuum flux at millimeter wavelengths increases toward the more evolved sources.

2. Through comparison to predictions from dust radiative transfer models, an upper limit on the contribution from protostellar envelope to the interferometric continuum flux is estimated - relative to the continuum flux measured on larger scales with single-dish telescopes. For the Class I sources about $87 \%$ of the observed SMA continuum flux (median value for the sample of Class I sources) has its origin in the circumstellar disks with the remainder coming from the large-scale envelope. For the Class 0 sources, $68 \%$ of the interferometric emission is related to the circumstellar disks.

3. Using empirical relationships between the envelope and disk fluxes and their masses from the radiative transfer models, the observations show that the disk-to-envelope mass ratio increases from about $1-10 \%$ in the Class 0 stage to about 20-60\% in the Class I stage, reflecting the dissipation of the envelope in the more evolved stages.
4. The compact interferometric fluxes due to disks are observed to decrease from the Class 0 to the Class I objects. However, this decrease is largely due to the changing physical properties, in particular disk temperature, during this phase. If these effects are taken into account, the disk mass shows no significant trends from the Class 0 to I stages and is typically $0.05 M_{\odot}$, albeit with significant scatter. Thus, no evidence is seen for significant disk mass growth from the Class 0 to the Class I stages.

5. Four Class I sources in the sample show evidence of Keplerian rotation in $\mathrm{HCO}^{+} 3-2$, and for IRS 43 also in HCN 3-2. IRS 63 and IRS 43 show evidence for elongated "continuum disks" at $1.1 \mathrm{~mm}$. For IRS 43 both the "continuum disk" and the $\mathrm{HCO}^{+} 3-2$ emission coincides with a dark lane in the HST $1.6 \mu \mathrm{m}$ images - and orientated perpendicular to the direction of the protostellar outflow. For the four Class I sources we derive central masses of $0.3-2.5 M_{\odot}$ from the Keplerian rotation curve.

6. The high excitation $\mathrm{HCO}^{+}$and $\mathrm{HCN}$ lines appear to be good tracers of the motions in the inner envelopes and disks, although in the most massive envelopes their use for this purpose is limited by the lines of the main isotopes being optical thick. Future observations with ALMA will allow similar studies using the less abundant isotopologues.

7. We compare the derived envelope and stellar masses to models for the mass evolution in a collapsing protostellar system including the formation and growth of a central circumstellar disk (Visser et al. 2009). The data are in agreement with phenomenological definition of Class 0 sources having accreted less, and Class I sources more, than half of their final mass. The models cannot explain the observed stellar masses for the handful of Class I sources for which these have been measured from the disk dynamical structure: generally the models produce too massive disks relative to their stellar masses.

8. Taken together these results suggest that circumstellar disks are established early in the evolution of low-mass protostars, but at the same time transport material rapidly from the larger scale envelopes onto the central stars. This may occur in episodic events, which may explain why the "luminosity problem" (that embedded YSOs are under-luminous compared to the theoretical predictions from pure accretion models) appears already during the Class 0 protostellar stages (e.g., Evans et al. 2009).

An important test of the theoretical models for the evolution of embedded young stellar objects will be to measure the disk dynamical structure even in the most deeply embedded stages. In this way it will be possible to address whether these disks have in fact settled into a rotationally stable pattern - and if so what central stellar masses are for the central newly formed star - or if the disk dynamics perhaps suggest some other configuration, for example, unstable "pseudo-disks". In this context, it remains a fundamental question whether the central stellar masses of the Class 0 objects indeed are significantly lower than those of the Class I sources, which would confirm their earlier evolutionary status.

Future ALMA observations will provide the sensitivity to molecules that are less abundant, and thus not severely optically thick in the outer envelopes, and thus a tracer of this structure. With ALMA it will also be possible to perform larger surveys of a statistically more unbiased sample of YSOs to constrain the evolution more directly. This will for example address whether traditional classification schemes for young stellar 
objects need to be recalibrated, whether the episodic accretion scenarios for YSOs are tied to the properties of their circumstellar disk, whether there are significant variations between accretion histories of protostars in different star forming environments, and what fraction of the envelope material is accreted onto the star and what fraction is carried away by the outflows.

Acknowledgements. We thank the referee for good suggestions about the presentation of these results. It is also a pleasure to thank the Submillimeter Array staff for help in carrying out observations and technical assistance with the resulting data. The Submillimeter Array is a joint project between the Smithsonian Astrophysical Observatory and the Academia Sinica Institute of Astronomy and Astrophysics, and is funded by the Smithsonian Institution and the Academia Sinica. This research was based in part on observations from the James Maxwell Telescope archive at the Canadian Astronomy Data Centre operated by the National Research Council of Canada with the support of the Canadian Space Agency. It is also used observations made with the NASA/ESA Hubble Space Telescope, obtained from the data archive at the Space Telescope Institute. STScI is operated by the association of Universities for Research in Astronomy, Inc. under the NASA contract NAS 5-26555. Astrochemistry in Leiden is supported by a Spinoza Grant from the Netherlands Organization for Scientific Research (NWO) and a NOVA grant. The research of T.L.B. was supported by NASA Origins grant NNX09AB89G.

\section{References}

Allen, L. E., Myers, P. C., Di Francesco, J., et al. 2002, ApJ, 566, 993

André, P., \& Montmerle, T. 1994, ApJ, 420, 837

André, P., Ward-Thompson, D., \& Barsony, M. 1993, ApJ, 406, 122

André, P., Ward-Thompson, D., \& Barsony, M. 2000, in Protostars and Planets IV, ed. V. Mannings, A. P. Boss, \& S. S. Russell (Tucson: University of Arizona Press), 59

Andrews, S. M., \& Williams, J. P. 2005, ApJ, 631, 1134

Andrews, S. M., \& Williams, J. P. 2007a, ApJ, 671, 1800

Andrews, S. M., \& Williams, J. P. 2007b, ApJ, 659, 705

Arce, H. G., \& Sargent, A. I. 2006, ApJ, 646, 1070

Barsony, M., Kenyon, S. J., Lada, E. A., \& Teuben, P. J. 1997, ApJS, 112, 109

Basu, S. 1998, ApJ, 509, 229

Beckwith, S. V. W., Sargent, A. I., Chini, R. S., \& Guesten, R. 1990, AJ, 99, 924

Brinch, C., Crapsi, A., Jørgensen, J. K., Hogerheijde, M. R., \& Hill, T. 2007, A\&A, 475, 915

Brinch, C., Jørgensen, J. K., \& Hogerheijde, M. R. 2009, A\&A, 502, 199

Brown, D. W., \& Chandler, C. J. 1999, MNRAS, 303, 855

Cassen, P., \& Moosman, A. 1981, Icarus, 48, 353

Chandler, C. J., Terebey, S., Barsony, M., Moore, T. J. T., \& Gautier, T. N. 1996, ApJ, 471, 308

Chiang, H.-F., Looney, L. W., Tassis, K., Mundy, L. G., \& Mouschovias, T. C. 2008, ApJ, 680, 474

Curiel, S., Girart, J. M., Rodríguez, L. F., \& Cantó, J. 2003, ApJ, 582, L109

D'Alessio, P., Calvet, N., \& Hartmann, L. 2001, ApJ, 553, 321

Di Francesco, J., Johnstone, D., Kirk, H., MacKenzie, T., \& Ledwosinska, E. 2008, ApJS, 175, 277

Duchêne, G., Bouvier, J., Bontemps, S., André, P., \& Motte, F. 2004, A\&A, 427, 651

Dullemond, C. P., \& Dominik, C. 2004, A\&A, 417, 159

Dunham, M. M., Crapsi, A., Evans, II, N. J., et al. 2008, ApJS, 179, 249

Eisner, J. A., Hillenbrand, L. A., Carpenter, J. M., \& Wolf, S. 2005, ApJ, 635, 396

Enoch, M. L., Glenn, J., Evans, II, N. J., et al. 2007, ApJ, 666, 982

Evans, N. J. 1999, ARA\&A, 37, 311

Evans, N. J., Dunham, M. M., Jørgensen, J. K., et al. 2009, ApJS, 181, 321

Galli, D., \& Shu, F. H. 1993a, ApJ, 417, 220

Galli, D., \& Shu, F. H. 1993b, ApJ, 417, 243

Girart, J. M., Rodríguez, L. F., \& Curiel, S. 2000, ApJ, 544, L153

Girart, J. M., Curiel, S., Rodríguez, L. F., et al. 2004, AJ, 127, 2969

Girart, J. M., Rao, R., \& Estalella, R. 2009, ApJ, 694, 56

Grosso, N., Alves, J., Neuhäuser, R., \& Montmerle, T. 2001, A\&A, 380, L1

Gutermuth, R. A., Myers, P. C., Megeath, S. T., et al. 2008, ApJ, 674, 336

Haisch, Jr., K. E., Greene, T. P., Barsony, M., \& Stahler, S. W. 2004, AJ, 127 1747

Hatchell, J., \& Fuller, G. A. 2008, A\&A, 482, 855

Hatchell, J., Fuller, G. A., Richer, J. S., Harries, T. J., \& Ladd, E. F. 2007, A\&A, 468, 1009

Ho, P. T. P., Moran, J. M., \& Lo, K. Y. 2004, ApJ, 616, L1

Hogerheijde, M. R., \& Sandell, G. 2000, ApJ, 534, 880
Hogerheijde, M. R., van Dishoeck, E. F., Blake, G. A., \& van Langevelde, H. J. 1997a, ApJ, 489, 293

Hogerheijde, M. R., van Langevelde, H. J., Mundy, L. G., Blake, G. A., \& van Dishoeck, E. F. 1997b, ApJ, 490, L99

Hogerheijde, M. R., van Dishoeck, E. F., Blake, G. A., \& van Langevelde, H. J. 1998, ApJ, 502, 315

Ivezić, Ž., Nenkova, M., \& Elitzur, M. 1999, User Manual for DUSTY, University of Kentucky Internal Report

Jørgensen, J. K. 2004, A\&A, 424, 589

Jørgensen, J. K., Schöier, F. L., \& van Dishoeck, E. F. 2002, A\&A, 389, 908 Jørgensen, J. K., Hogerheijde, M. R., van Dishoeck, E. F., Blake, G. A., \& Schöier, F. L. 2004a, A\&A, 413, 993

Jørgensen, J. K., Schöier, F. L., \& van Dishoeck, E. F. 2004b, A\&A, 416, 603 Jørgensen, J. K., Bourke, T. L., Myers, P. C., et al. 2005a, ApJ, 632, 973 Jørgensen, J. K., Lahuis, F., Schöier, F. L., et al. 2005b, ApJ, 631, L77 Jørgensen, J. K., Harvey, P. M., Evans, N. J. I., et al. 2006, ApJ, 645, 1246 Jørgensen, J. K., Bourke, T. L., Myers, P. C., et al. 2007a, ApJ, 659, 479 Jørgensen, J. K., Johnstone, D., Kirk, H., \& Myers, P. C. 2007b, ApJ, 656, 293 Jørgensen, J. K., Johnstone, D., Kirk, H., et al. 2008, ApJ, 683, 822 Keene, J., \& Masson, C. R. 1990, ApJ, 355, 635

Kenyon, S. J., Hartmann, L. W., Strom, K. M., \& Strom, S. E. 1990, AJ, 99, 869

Lay, O. P., Carlstrom, J. E., Hills, R. E., \& Phillips, T. G. 1994, ApJ, 434, L75 Leous, J. A., Feigelson, E. D., André, P., \& Montmerle, T. 1991, ApJ, 379, 683 Lommen, D., Jørgensen, J. K., van Dishoeck, E. F., \& Crapsi, A. 2008, A\&A, 481,141

Looney, L. W., Mundy, L. G., \& Welch, W. J. 2000, ApJ, 529, 477

Looney, L. W., Mundy, L. G., \& Welch, W. J. 2003, ApJ, 592, 255

Looney, L. W., Tobin, J. J., \& Kwon, W. 2007, ApJ, 670, L131

Montmerle, T., Grosso, N., Tsuboi, Y., \& Koyama, K. 2000, ApJ, 532, 1097

Mundy, L. G., Looney, L. W., Erickson, W., et al. 1996, ApJ, 464, L169

Myers, P. C., \& Ladd, E. F. 1993, ApJ, 413, L47

Myers, P. C. 2008, ApJ, 687, 340

Ossenkopf, V., \& Henning, T. 1994, A\&A, 291, 943

Padgett, D. L., Rebull, L. M., Stapelfeldt, K. R., et al. 2008, ApJ, 672, 1013

Qi, C. 2008, The MIR Cookbook, The Submillimeter Array / Harvard-Smithsonian Center for Astrophysics (http: //cfa-www . harvard. edu/ cqi/mircook.html)

Sault, R. J., Teuben, P. J., \& Wright, M. C. H. 1995, in Astronomical Data Analysis Software and Systems IV, ed. R. A. Shaw, H. E. Payne, \& J. J. E. Hayes, PASP Conf. Ser., 77, 433

Schöier, F. L., Jørgensen, J. K., van Dishoeck, E. F., \& Blake, G. A. 2002, A\&A, 390,1001

Schöier, F. L., Jørgensen, J. K., van Dishoeck, E. F., \& Blake, G. A. 2004, A\&A, 418,185

Shirley, Y. L., Evans, N. J., \& Rawlings, J. M. C. 2002, ApJ, 575, 337

Shu, F. H. 1977, ApJ, 214, 488

Stutz, A. M., Rubin, M., Werner, M. W., et al. 2008, ApJ, 687, 389

Tassis, K., \& Mouschovias, T. C. 2005, ApJ, 618, 783

Terebey, S., Shu, F. H., \& Cassen, P. 1984, ApJ, 286, 529

Terebey, S., Chandler, C. J., \& Andre, P. 1993, ApJ, 414, 759

Terebey, S., van Buren, D., Padgett, D. L., Hancock, T., \& Brundage, M. 1998, ApJ, 507, L71

Terebey, S., Van Buren, D., Matthews, K., \& Padgett, D. L. 2000, AJ, 119, 2341

Tobin, J. J., Hartmann, L., Calvet, N., \& D’Alessio, P. 2008, ApJ, 679, 1364

Tsuboi, Y., Imanishi, K., Koyama, K., Grosso, N., \& Montmerle, T. 2000, ApJ, 532, 1089

Ulrich, R. K. 1976, ApJ, 210, 377

van der Tak, F. F. S., Black, J. H., Schöier, F. L., Jansen, D. J., \& van Dishoeck, E. F. 2007, A\&A, 468, 627

van Kempen, T. A., van Dishoeck, E. F., Salter, D. M., et al. 2009, A\&A, 498, 167

Velusamy, T., \& Langer, W. D. 1998, Nature, 392, 685

Visser, R., van Dishoeck, E. F., Doty, S. D., \& Dullemond, C. P. 2009, A\&A, 495,881

Vorobyov, E. I., \& Basu, S. 2006, ApJ, 650, 956

Ward-Thompson, D. 2007, Joint 32nd International Conference on Infrared and Millimeter Waves, and 15th International Conference on Terahertz Electronics. IRMMW-THz, ed. M. Griffin, P. C. Hargrave, T. J. Parker, \& K. P. Wood, 720

Weintraub, D. A., Kastner, J. H., Griffith, L. L., \& Campins, H. 1993, AJ, 105, 271

Yorke, H. W., \& Bodenheimer, P. 1999, ApJ, 525, 330

Young, E. T., Lada, C. J., \& Wilking, B. A. 1986, ApJ, 304, L45

Young, C. H., Shirley, Y. L., Evans, N. J., \& Rawlings, J. M. C. 2003, ApJS, 145, 111

Young, K. E., Enoch, M. L., Evans, II, N. J., et al. 2006, ApJ, 644, 326

Zhang, Q., Wootten, A., \& Ho, P. T. P. 1997, ApJ, 475, 713 


\section{Appendix A: The use of $\mathrm{HCO}^{+}$as a dense gas tracer}

\section{A.1. Outflow cavities in TMR1, TMC1A and GSS30-IRS1}

The $\mathrm{HCO}^{+} 3-2$ alignment with the near-infrared scattering nebulosities in the HST images for TMR1, TMC1A and GSS30-IRS1 (Fig. 10) is similar to what has been found in high angular resolution observations of the lower excitation 1-0 transition in both Class I (Hogerheijde et al. 1997a) and Class 0 (Jørgensen 2004) sources.

The patterns in the velocity maps (Fig. 11) support the interpretation that the $\mathrm{HCO}^{+} 3-2$ emission has its origin in one-side of the outflow cone: in all maps the $\mathrm{HCO}^{+}$emission is blueshifted with respect to the systemic velocity with the most extreme velocities (most blue-shifted) narrowly confined around the continuum position and material closer to the systemic velocity extending around this. This signature can best be understood in a simple geometry where $\mathrm{HCO}^{+}$probes material in a large infalling protostellar envelope being swept up by the outflow, as illustrated in Fig. A.1. If the velocities in the swept-up material are relatively small (comparable to the infalling velocities in the envelope) and $\mathrm{HCO}^{+} 3-2$ optically thick, the front-side of the envelope (toward us) will be slightly red-shifted due to the infall and thus obscure the outflowing material in the red-shifted cone on the far-side of the envelope (Fig. A.1), which in turn is resolved-out. In contrast, the outflow cone pointed toward us will be unobscured by the envelope. Likewise, the near-infrared scattered light from the red-lobe would largely be blocked - in contrast to the scattered emission from the outflow cavity on the front-side. An alternative explanation is that all the sources are at the far-side of their respective clouds, the red-shifted part of the outflow cone breaking out into a low-density medium not observable in the line tracers. This would require a rather sharp density gradient, however.

\section{A.2. Limitations of $\mathrm{HCO}^{+} 3-2$ as a tracer of disks}

The results above suggest that $\mathrm{HCO}^{+} 3-2$ is a good tracer of motions in disks for envelopes that have already been largely dispersed. For the more embedded $\mathrm{YSO}_{\mathrm{S}} \mathrm{HCO}^{+} 3-2$ emission may be of limited use as a tracer of rotation in the disk due to its optical thickness. A simple estimate can be made of the typical envelope mass below which $\mathrm{HCO}^{+} 3-2$ line emission is optically thin in the envelope and can be used as a tracer of the kinematics in the innermost regions of the protostar. First, for typical envelope parameters we expect temperatures in the range of 15-40 K and densities of $10^{4}-10^{7} \mathrm{~cm}^{-3}$. Using the Radex escape probability code (van der Tak et al. 2007) ${ }^{3}$ for these parameters, the $\mathrm{HCO}^{+}$ 3-2 line has an optical thickness $\approx 1$ for an $\mathrm{HCO}^{+}$column density of $5 \times 10^{12} \mathrm{~cm}^{-2}$ and a line width of $1 \mathrm{~km} \mathrm{~s}^{-1}$. Secondly, we can estimate what density, $n_{100} \mathrm{AU}$ is required to reach that optical thickness in a free-falling envelope with a power-law density profile, $n=(r / 100 \mathrm{AU})^{-1.5}$ at a size scale of $100 \mathrm{AU}$ corresponding to the region where the disk potentially forms, i.e., by simply estimating $n_{100} \mathrm{AU}$ from

$N_{\mathrm{HCO}+}=\int_{10^{2} \mathrm{AU}}^{10^{4} \mathrm{AU}} n_{100 \mathrm{AU}}(r / 100 \mathrm{AU})^{-1.5} \mathrm{~d} r$

with $\mathrm{N}_{\mathrm{HCO}+}=5 \times 10^{12} \mathrm{~cm}^{-2}$. Assuming a typical abundance of $\mathrm{HCO}^{+}$of $1 \times 10^{-9}$ with respect to $\mathrm{H}_{2}$ (e.g., Jørgensen et al. $2004 \mathrm{~b}$ ), we find $n_{100 \mathrm{AU}}=2 \times 10^{6} \mathrm{~cm}^{-3}$, which in turn corresponds to an envelope mass of $0.1 M_{\odot}$. This is the maximum

${ }^{3}$ http://www.sron.rug.nl/ vdtak/radex/radex.php

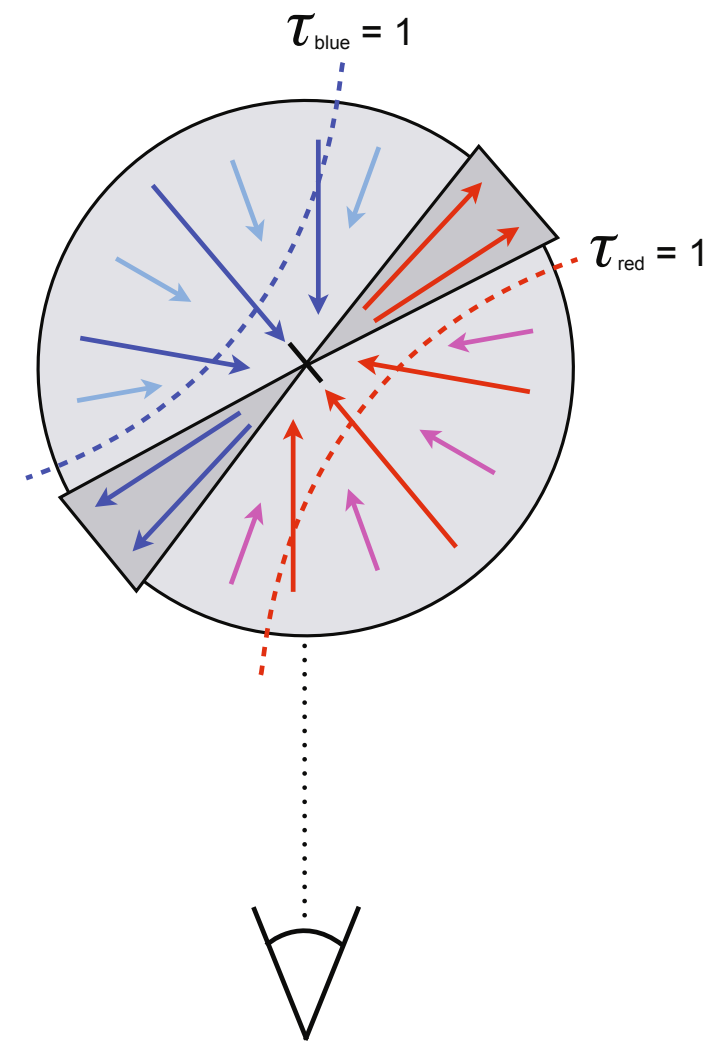

Fig. A.1. Schematic figure of a collapsing envelope with an outflow sweeping up material. The dashed lines indicate the $\tau=1$ surfaces for a red- and blue-shifted velocity ( $\tau_{\text {red }}$ and $\tau_{\text {blue }}$, respectively) for a line which becomes optically thick at low column densities through the envelope. As illustrated in the figure, the blue-shifted part of the outflow cone may appear in front of the $\tau_{\text {blue }}=1$ surface, while the red-shifted part is behind the $\tau_{\text {red }}=1$ surface, obscured by material in the large scale collapsing envelope, which in turn may be resolved out by the interferometer.

envelope mass for which we can use the $\mathrm{HCO}^{+} 3-2$ to trace scales smaller than about 100 AU without worrying about opacity effects in the larger scale envelope. It indeed corresponds to the estimate of the masses of the most deeply embedded Class I sources in our sample for which the outflow emission is seen. For these sources and the even more deeply embedded Class 0 sources a less abundant or more optically thin tracer is therefore required to trace the dynamical structure on less than $100 \mathrm{AU}$ scales. Future observations with ALMA will provide the sensitivity to image, e.g., the optically thin 3-2 and 4-3 submillimeter transitions of $\mathrm{H}^{13} \mathrm{CO}^{+}$in the disks around even more deeply embedded protostars and thus provide stellar masses for protostars in the deeply embedded stages.

\section{Appendix B: Summary of observed Class I sources}

\section{B.1. IRS 43}

IRS 43 (YLW15; Young et al. 1986) has been the target of numerous studies across the full wavelength range. At radio wavelengths, IRS 43 is resolved into two separate components, YLW15-VLA1 and -VLA2 with a separation of 0.6" (Girart et al. 2000). From multi-epoch high angular resolution radio data, these binary components were found to show relative proper motions indicative of orbital motions in a $1.7 M_{\odot}$ total 
mass binary (Curiel et al. 2003). Girart et al. found that one of the two components, VLA1, were resolved in observations at 3.6 and $6 \mathrm{~cm}$ with the VLA with a deconvolved size of about $0.4^{\prime \prime}$ along its major axis and suggested that it was associated with a thermal radio jet. In addition, IRS 43 is a peculiar X-ray emitter showing quasi-periodic X-ray flares (Tsuboi et al. 2000) consistent with magnetic shearing and reconnection between the central star and an accretion disk, with the period suggesting a mass of the central star in the range 1.8-2.2 $M_{\odot}$ (Montmerle et al. 2000).

The region was imaged in the near-infrared by Grosso et al. (2001) who discovered a set of "embedded" HerbigHaro objects, well aligned with the thermal jet candidate from the centimeter observations of Girart et al. (2000). Spitzer Space Telescope images of the embedded Herbig-Haro objects (Fig. B.1) show the knots clearly along a line pointing directly back toward the group of objects where IRS 43 and another embedded protostar, IRS 44, is located. As also pointed out by Grosso et al., the association of the Herbig-Haro knots to IRS 43 is not unique: the nearby protostar, IRS 54 (see also Sect. B.5), also drives a large-scale, precessing outflow clearly seen in the Spitzer images in Fig. B.1.

The dotted lines in Fig. B.1 show the propagation of the IRS 54 outflow, following the $\mathrm{H}_{2}$ emission in the eastern lobe and assuming that the precession of the IRS 54 outflow is symmetric around the protostar - thereby giving the expected location of the western lobe. The western lobe in this case passes through the location of the southern part of the near-infrared Herbig-Haro objects: based on their morphologies in the higher resolution near-infrared images, Grosso et al. (2001) suggested that these knots (crosses in the lower left panel of Fig. B.1) are indeed caused by the IRS 54 outflow with the northern knots (plus signs in the lower left panel of Fig. B.1 related to the IRS 43 outflow - the lower spatial resolution Spitzer images do not reveal this distinction clearly, though. Some support for the interpretation of Grosso et al. can be taken from the morphology of another bow-shock seen in the Spitzer images at $(\alpha, \delta)_{\mathrm{J} 2000}=\left(16^{\mathrm{h}} 27^{\mathrm{m}} 39.5^{\mathrm{s}},-24^{\circ} 33^{\prime} 02^{\prime \prime}\right)$ to the west of the near-infrared Herbig-Haro knots. Together with the nearinfrared Herbig Haro knots detected by Grosso et al., this bowshock is on the southern side of the dashed-line suggesting that the dashed line indeed is delineating the northern edge of the western lobe (and the southern edge of the eastern lobe) of the IRS 54 outflow. It is also possible that another source in the group encompassing IRS 43 could be responsible for the Herbig-Haro knots: the main argument for the association to IRS 43 is the coincidence with the radio jet and the identification of a bow-shock south of IRS 43 in the near-infrared, possibly representing the counter-jet (Grosso et al. 2001; Girart et al. 2000, 2004).

We detect IRS 43 in both $\mathrm{HCO}^{+} 3-2$, $\mathrm{HCN} 3-2$ and continuum. The $\mathrm{HCN}$ and $\mathrm{HCO}^{+} 3-2$ emission is elongated in the $\mathrm{W} / \mathrm{NW}-\mathrm{E} / \mathrm{SE}$ direction with a clear velocity gradient along its major axis. The major axis of the $\mathrm{HCO}^{+}$and $\mathrm{HCN} 3-2$ emission is close to the axis of the continuum emission - and perpendicular to the direction toward the Herbig-Haro objects (Fig. B.1; Grosso et al. 2001) and the thermal continuum jet (Girart et al. 2000).

\section{B.2. $T M R 1$ and TMC1A}

Images of TMR1 using the HST/NICMOS at 1.6-2.1 $\mu \mathrm{m}$ by Terebey et al. (1998) show s triple system consisting of a close binary (TMR-1A and TMR-1B) with a projected separation of $0.3^{\prime \prime}$ and a third source TMR-1C offset from the binary by about $10^{\prime \prime}$ but connected with this through an extended filament of near-infrared nebulosity. Terebey et al. (1998, 2000) discuss the option that TMR-1C is a runaway giant planet, but conclude based on near-infrared spectroscopy that it is most likely is a background star. TMC1A is an embedded protostar with an associated scattering nebulosity (e.g., Chandler et al. 1996; Hogerheijde et al. 1998) aligned with blue-shifted outflow emission.

Both TMR1 and TMC1A are detected in continuum, together with $\mathrm{HCO}^{+}$and $\mathrm{HCN} 3-2$. Both show clear evidence of the line emission being offset from the continuum peaks toward the outflow nebulosity observed in the near-infrared HST/NICMOS images - similar to the case for GSS30-IRS1. The offsets in the $\mathrm{HCO}^{+} 3-2$ emission toward the two sources are similar to those observed in $\mathrm{HCO}^{+} 1-0$ by Hogerheijde et al. (1998).

\section{B.3. GSS30}

The GSS30 region is the most complex of the studied fields associated with a clear near-infrared nebulosity. At least three young stellar objects (GSS30-IRS1, -IRS2 and -IRS3) are seen at infrared wavelengths, located within the SMA primary beam field of view, (Weintraub et al. 1993) and observed by Spitzer (e.g., Jørgensen et al. 2008). GSS30-IRS1 is located at the center of this outflow cone and has a mid-infrared SED slope consistent with a Class I protostar. GSS 30-IRS3 (LFAM1) was detected in high-resolution images at $2.7 \mathrm{~mm}$ (Zhang et al. 1997) and $6 \mathrm{~cm}$ (Leous et al. 1991). GSS30-IRS2 is likely a more evolved (T Tauri) young stellar object also detected at $6 \mathrm{~cm}$.

The SMA continuum observations identify one protostar GSS30-IRS3 (LFAM1) whereas the two other protostars remain undetected down to the noise level of $\approx 3 \mathrm{mJy} \mathrm{beam}^{-1}$. The same was the case for the similar in the $2.7 \mathrm{~mm}$ observations by Zhang et al. (1997). The single-dish continuum emission toward GSS30 is often attributed solely to GSS30-IRS1 (e.g., Zhang et al. 1997), but appears to be peaking at GSS30-IRS3 consistent with the suggestion that this source is more deeply embedded.

The $\mathrm{HCO}^{+}$line emission shows a distinct peak at the location of GSS30-IRS1 with a narrow line detected toward GSS30-IRS2 (Fig. 4). HCN is also clearly detected toward GSS30, again toward GSS30-IRS1, but offset from the $\mathrm{HCO}^{+} 3-2$ emission. Toward IRS1, both $\mathrm{HCO}^{+}$and $\mathrm{HCN}$ appear to trace one side of the outflow cone directed toward us.

\section{B.4. WL 12}

WL 12 is a "standard" Class I YSO: it is neither a binary, nor does it have a spectacular outflow. It is clearly detected in the interferometric continuum data, but not in the $\mathrm{HCO}^{+}$or HCN 3-2 maps. It is identified as a core in SCUBA maps and included in the list of embedded YSOs by Jørgensen et al. (2008) with red mid-infrared colors characteristic of those sources, as well as bright $\mathrm{HCO}^{+} 4-3$ emission (van Kempen et al. 2009).

\section{B.5. IRS 54}

IRS 54 is found to be a binary with a separation of about $7^{\prime \prime}$ in deep near-infrared wavelength observations (Haisch et al. 2004; Duchêne et al. 2004), possibly also responsible for the precession of the outflow observed in the mid-infrared Spitzer images (Fig. B.1). IRS 54 is the only source in our sample, which is 

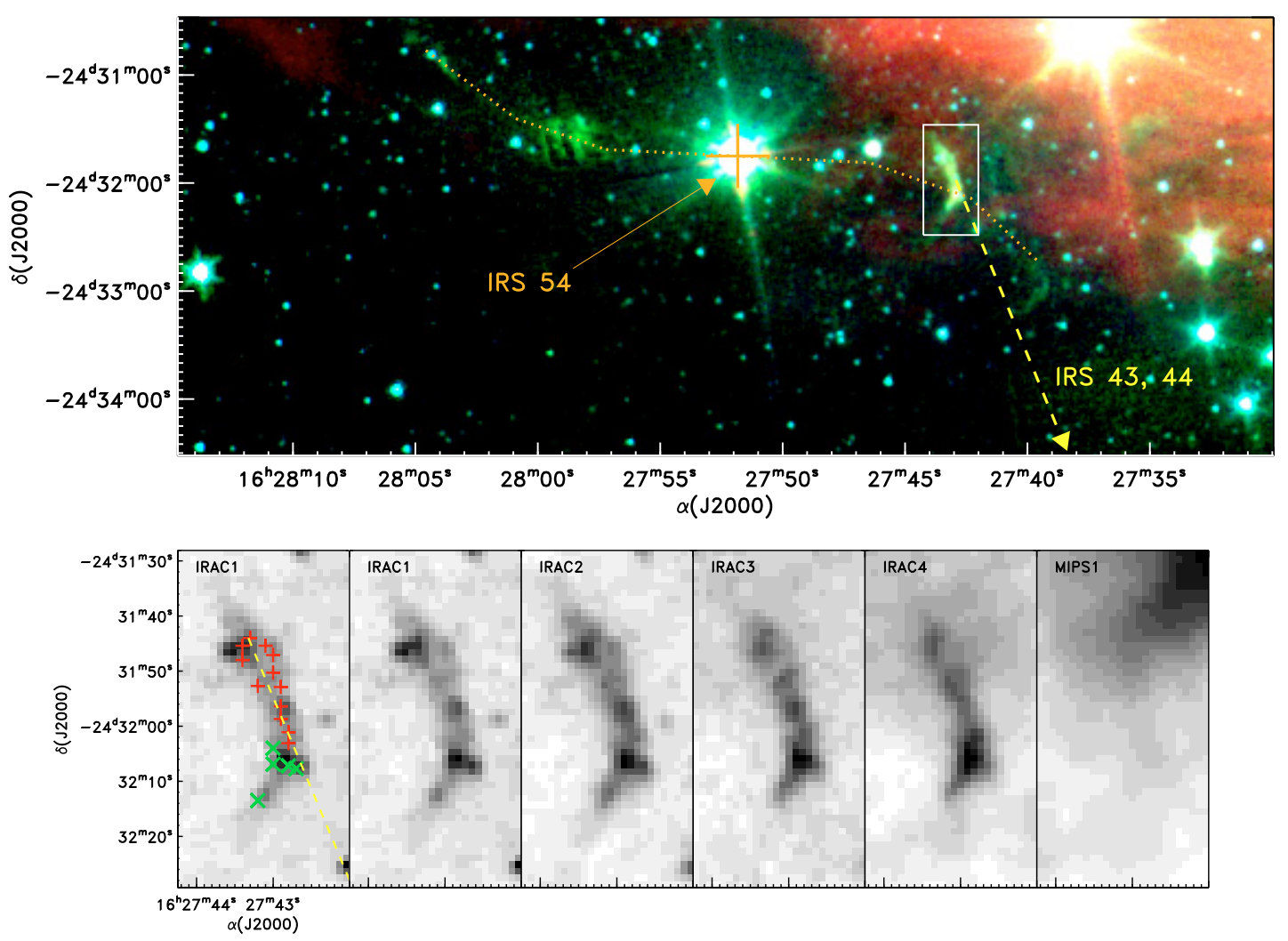

Fig. B.1. Upper panel: the region of the IRS 54 outflow as well as the near-infrared Herbig-Haro objects from Spitzer observations with $3.6 \mu \mathrm{m}$ in blue, $4.5 \mu \mathrm{m}$ in green and $8.0 \mu \mathrm{m}$ in red. In addition we show the suggested direction of the precessing IRS 54 outflow (dotted line) and the direction to IRS 43 and IRS 44 (dashed arrow). Lower panels: images of the near-infrared Herbig-Haro knots from 3.6-8.0 $\mu$ m (IRAC1-4) and $24 \mu \mathrm{m}$ (MIPS1). The location of the Herbig-Haro knots suggested by Grosso et al. (2001) to be associated with the IRS 54 outflow are shown with the crosses, whereas the knots proposed to be associated with the IRS 43 outflow are shown with plus-signs in the first panel.

not detected in either continuum and only shows a tentative detection in $\mathrm{HCO}^{+} 3-2$. It is associated with a faint SCUBA core (peak of $0.1 \mathrm{Jy} \mathrm{beam}^{-1}$; total integrated flux in a $40^{\prime \prime}$ radius aperture of $0.4 \mathrm{Jy}$ ) below the threshold for core detection in Jørgensen et al. (2008). It is also not identified as a separate millimeter core in the Bolocam maps of Young et al. (2006). Based on its $\mathrm{HCO}^{+} 4-3$ line emission, van Kempen et al. (2009) classify it as a "late Stage 1" source in transition to the T Tauri stage.

\section{B.6. IRS 63 and Elias 29}

The results for IRS 63 and Elias 29 are discussed in more detail in Lommen et al. (2008). In summary, the two sources are detected in both continuum and $\mathrm{HCO}^{+} 3-2$. IRS 63 shows the strongest continuum emission by about a factor 8 whereas the Elias 29 is stronger in $\mathrm{HCO}^{+} 3-2$ also by about a factor 8 . Where Elias 29 clearly is associated with strong extended continuum emission, witnessed both by its brightness profile in the SMA data and by comparison to single-dish observations, IRS 63 is dominated by emission from a compact, unresolved component, consistent with the suggestion that it is a source in transition between the embedded and $\mathrm{T}$ Tauri stages with $M_{\mathrm{env}} \sim M_{\mathrm{disk}}$. 